\title{
Sediment transport and trench development beneath a cylinder oscillating normal to a sandy seabed
}

\author{
Joe G. Tom ${ }^{\mathrm{a}, \mathrm{b}, *}$, Scott Draper ${ }^{\mathrm{a}, \mathrm{b}}$, David J. White ${ }^{\mathrm{a}, \mathrm{c}}$ \\ ${ }^{a}$ Centre for Offshore Foundation Systems, The University of Western Australia, Crawley 6009, WA, Australia \\ ${ }^{b}$ Ocean Graduate School, The University of Western Australia, Crawley 6009, WA, Australia \\ ${ }^{c}$ Faculty of Engineering and Physical Sciences, University of Southampton, Southampton SO17 1BJ, United Kingdom
}

\begin{abstract}
The purpose of this study is to explore the conditions in which trenches form beneath oscillating cylinders such as pipelines, cables or idealised chains - close to the seabed. Experiments are conducted by oscillating a circular cylinder in a direction normal to an initially flat sandy bed. Across a relatively wide parameter space, the transport patterns and trench geometries reveal three transport regimes that are linked to vortex dynamics and depend primarily on the ratio of oscillation amplitude to cylinder diameter ( $K C$ number). For $K C \lesssim 4$ sediment motion results in bedload transport that is symmetric about the cylinder centreline. This leads to the formation of two parallel trenches with a prominent ridge forming directly beneath the cylinder. For $4 \lesssim K C \lesssim 9$ sediment motion occurs via localised transport events, which are associated with the motion of vortices shed from the cylinder. These transport events are irregular but occur on both sides of the cylinder and lead to the formation of a symmetric trench geometry. For $9 \lesssim K C \lesssim 12$ the sediment motion is characterised by localised transport events and asymmetric bedload transport driven by overall vortex dynamics. In terms of trench size, the maximum (equilibrium) depth is found to increase with $K C$ and a mobility number $(\psi)$ defined in terms of the maximum cylinder velocity. The initial rate of trench development also increases with $K C$ number and $\psi$, with an additional dependency on the cylinder Reynolds number. The cylinder motions required to initiate trenching are predicted well using continuity arguments and an oscillatory boundary layer assumption, provided the $K C$ number and minimum gap between the cylinder and the bed are relatively small. The findings in this study provide insight into the mechanisms and prediction of trench formation. In particular, this study reveals that significant trenches can form in sandy seabeds solely due to fluid flow induced by pipeline/cable/chain motion without direct seabed contact, which has implications for structural fatigue.
\end{abstract}

Keywords: Pipelines; risers; sediment transport; trenching; chains; cables

\footnotetext{
${ }^{*}$ Corresponding author.

Email address: joe.tom@uwa.edu.au (Joe G. Tom)
} 


\section{Introduction}

Objects that oscillate above the seabed cause local fluid motions that have the potential to cause sediment transport even without the influence of externally-applied flows. This transport can result in the formation of a trench beneath the object, which can impact the design of engineered near-seabed infrastructure in the offshore industry. For example, full utilisation of the fatigue life of steel catenary risers (SCRs) in deep-water offshore developments is limited by the inability to predict the seabed geometry due to oscillation of the riser at the touchdown zone (Bridge and Howells, 2007). This has implications on how the stresses in the riser vary close to the touchdown point over the lifetime of the system. Similarly, the significant trenches believed to result from soil-fluid-structure interaction around mooring line chains can significantly reduce the capacity of anchoring systems (Bhattacharjee et al., 2014). For both SCRs and mooring lines, vertical oscillation in absence of background flows occurs if metocean loading on connected surface facilities is independent of the near-bottom metocean climate - for instance surface waves in deep water environments. Similarly, isolated near-bottom motion occurs during installation of pipelines and cables from surface pipe-lay vessels (Westgate et al., 2010a).

Despite the practical importance of trench formation, limited previous work has studied the mechanics of scour owing solely to object motion. Chiew et al. (2016) performed an experimental study describing trench geometries that form in a sandy bed beneath a spring-mounted cylinder undergoing forced vibrations normal to the bed and allowed to contact the bed. The study focused on two oscillation frequencies and a targeted oscillation amplitude of 1.4 diameters. For these two cases the observed flow field was reported to be symmetric and the trench that formed was symmetric about the centreline of the cylinder. Interestingly, the maximum width of the trench was found to increase with increasing oscillation frequency, whilst the maximum depth decreased with increasing oscillation frequency. Chiew et al. (2016) provided important insight into trenching mechanics, motivating the wider parametric range of the current experiments.

In contrast, there exists a substantial body of work regarding scour beneath pipelines due to externally applied hydrodynamic forcing, such as currents and/or waves, as opposed to object motion (e.g. Sumer and Fredsøe, 2002). The basic features of wave-induced pipeline scour are (a) that the equilibrium scour depth is a function of the Keulegan-Carpenter $(K C)$ number, which is proportional to the orbital amplitude that a fluid particle moves during a cycle relative to the pipeline diameter; and (b) the time scale of scour formation is inversely proportional to the dimensionless skin friction shear stress applied to the seabed due to the wave forcing. Previous studies have also focused on scour beneath a vibrating pipeline or riser in the presence of currents or waves, which has relevance to vortex-induced vibrations (Sumer et al., 1988; Gao et al., 2006; Chiew et al., 2014). These and similar studies found that the scour depth generally increases with pipeline oscillation (relative to the stationary case) near the bed.

Based on classical fluid mechanics, it is well-known that if the oscillation amplitude of an object is 
not small compared with its diameter, vortex shedding may significantly influence the local flow field. Williamson (1985) and Tatsuno and Bearman (1990) documented the vortex shedding regimes around a cylinder oscillating far from any boundary. Williamson (1985) described the changes in flow regime that are observed as $K C$ number increases (where the $K C$ number is now proportional to the amplitude of cylinder motion divided by cylinder diameter), for $\beta=R e / K C<230$, where $R e$ is the Reynolds number defined in terms of the maximum cylinder velocity. At $K C<4$ a pair of vortices with approximately equal strength form on the trailing side of the cylinder and do not shed, but detach at the end of each half cycle. As $K C$ increases to $\sim 7$, the strength and size of the vortex pair become increasingly asymmetric, but do not shed due to insufficient amplitude of motion. Above $K C \sim 7$, one vortex sheds per half cycle leading to the formation of a vortex street transverse to the axis of oscillation. At higher $K C$ numbers, additional vortices are able to shed per half cycle, leading to a family of additional flow regimes (Sumer and Fredsøe, 2006).

With the introduction of a rigid wall, a third parameter (in addition to the $K C$ and $\beta$ numbers) influences the local flow field: the minimum gap distance between the cylinder and the wall. Sumer et al. (1991), for example, has shown that the transverse vortex street transitions to a parallel vortex street when a cylinder is oscillated parallel and sufficiently close to a wall. Sumer et al. (1991) also showed that vortex shedding is suppressed at small gap distances (less than 0.1D). For the case of a cylinder oscillating normal to a rigid wall, Tom (2018), using particle image velocimetry experiments, showed that at low $K C$ ( $\lesssim 5$ but also up to at least 12 for some portions of the cycle) and $\beta=500$ the presence of a wall leads to relatively large near-boundary velocities (or pumping) that can be predicted well by potential theory and continuity arguments. For $K C \gtrsim 7$ vortices shed in the transverse direction but the confinement introduced by the wall causes horizontal flows near the wall to be strongly asymmetric. The influence of vortices was also suggested to increase the near-wall velocity, relative to that for symmetric pumping, for $K C \gtrsim 5$. Tom (2018) also showed that for a minimum gap height of $12.5 \%$ of the cylinder diameter, the wall does not appear to suppress transverse motion of shed vortices from a normally oscillating cylinder. The numerical results presented by Tom (2018) over this $K C$ range focused on a constant $R e=150$. The results showed similar vortex-wall interaction features to the experimental results for larger $R e$ and showed that comparison between continuity arguments and near-wall velocity is good provided the minimum gap ratio and $K C$ number are sufficiently small.

Building on this previous body of research, the principle aim of this paper is to experimentally investigate trench formation beneath an oscillating cylinder over a relatively wide parameter space that is relevant to submarine pipelines, cables and mooring chains during installation and operation. As an idealisation of a typical riser system, a circular cylinder is oscillated sinusoidally in time normal to the bed (see Figure 1). A focus is placed on relating general observations of the trenching processes back to measurements of the velocity field obtained by Tom (2018) to elucidate the driving mechanisms of sediment transport. Three aspects of the problem are investigated quantitatively: (i) the range of cylinder motions that cause sediment 


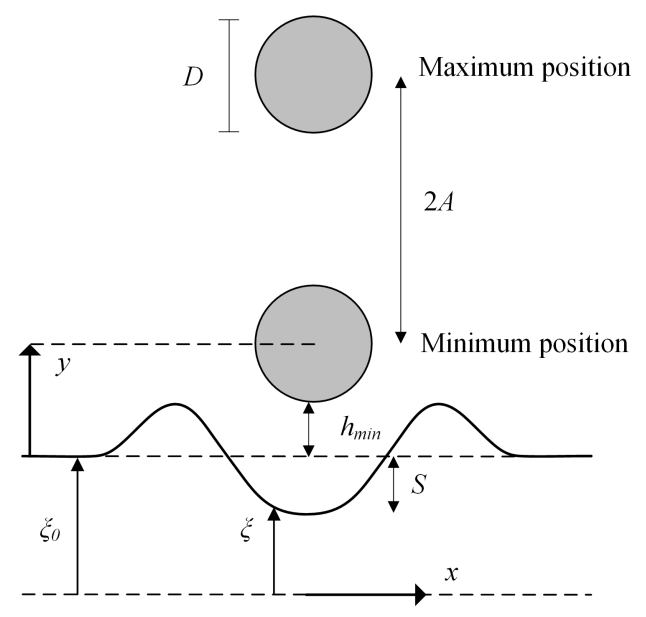

Fig. 1. Problem definition.

transport, to provide a prediction on when trenching may occur; (ii) the rate of trench formation and (iii) the extent and depth of the trench as it progresses.

To investigate the mechanics of trench formation due to fluid motions alone, the experiments reported in this paper are for cylinder motions that do not contact the bed. The geotechnical aspects of trench formation due to pipe-seabed contact are thus avoided. Similarly, the present experiments only use sandy, coarsegrained sediment. This restriction allows for interpretation of trench formation from the well-established standpoint of sand transport. It is anticipated that future work considering finer grain sediments may utilise the insights gained from the present experiments.

The remainder of this paper is structured as follows. Section 2 describes the experimental setup utilised and Section 3 outlines the relevant parameter space that has been investigated. Section 4 then presents an overview of the sediment transport mechanisms and the trench geometries that were observed across the experimental parameter space. The effect of cylinder motions on the initiation, rate and depth of trench formation are then quantitatively investigated in Section 5. Discussions and conclusions are presented in Section 6 and 7 , respectively.

\section{Experimental methodology}

The experiments were conducted in a $660 \mathrm{~mm}$ long section of a $395 \mathrm{~mm}$ wide tank at the University of Western Australia, which has a total length of $15 \mathrm{~m}$. The water depth was $250 \mathrm{~mm}$ above the bed surface and the sand bed was placed to a total depth of $200 \mathrm{~mm}$. The cylinder was oscillated using an electrical actuator whereby the prescribed vertical displacement in time was:

$$
y(t)=h_{\min }+\frac{D}{2}+A-A \cos \left(\frac{2 \pi t}{T}\right)
$$


Table 1

Properties of sediment used in experiments.

\begin{tabular}{l|l|l}
\hline Property & Units & Value \\
\hline Median particle size, $d_{50}$ & $(\mathrm{~mm})$ & 0.180 \\
Coefficient of uniformity, $C_{u}$ & $(-)$ & 2.000 \\
Particle specific gravity, $s$ & $(-)$ & 2.670 \\
Critical Shields parameter, $\theta_{c r}$ & $(-)$ & 0.051 \\
Critical shear stress for erosion, $\tau_{c r}$ & $(\mathrm{~Pa})$ & 0.159 \\
\hline
\end{tabular}

\section{Experimental parameter space} SS2).

where $A$ is the oscillation amplitude, $T$ is the period and $h_{\min }(>0)$ is the minimum gap distance. Since the motion is defined by a cosine function, the lowest point in the cycle occurs at $t / T=0,1,2, \ldots$ and the cylinder is at its furthest position at $t / T=0.5,1.5, \ldots$ To verify the cylinder motion, a linearly variable differential transformer was used to measure the cylinder displacement.

Three PVC cylinders were used (with diameters of $21 \mathrm{~mm}, 51 \mathrm{~mm}$ and $89 \mathrm{~mm}$ ), which extended $385 \mathrm{~mm}$ across the width of tank. Additional tests were also conducted with $650 \mathrm{~mm}$ long cylinders (extending along the tank section) to investigate end effects. Prior to each experiment, the sediment was levelled by dragging a thin, rectangular piece of PVC material over the test bed. The leveller extended across the width of the tank and was suspended below two railings placed atop the sediment container. The initial bed surface, denoted $\xi(x, z, 0)$, was then scanned using a SICK Ranger 3-D laser scanner. During testing cylinder motion was stopped at intervals and the bed was rescanned to recover $\xi(x, z, t)$. Scans were taken with the laser placed across the width of the tank and actuated along its length.

The sediment used in the experiments was commercially available silica sand, which has been employed in a number of previous scour studies (e.g. Leckie et al., 2016). Table 1 presents the properties of the sediment drawing on erosion parameters determined by Mohr et al. (2016) (who refer to this sediment as

Dimensional considerations indicate that trench development beneath the cylinder is dependent on a number of variables related to the cylinder motion, fluid properties and sediment properties. The maximum trench depth $S_{0}=\xi_{0}-\min (\xi)$, for example, may be written functionally as:

$$
S_{0}=f\left(D, A, T, h_{\text {min }}, \nu, d_{50}, g(s-1), w_{s}\right)
$$

where $\nu$ is the fluid kinematic viscosity and $d_{50}$ is the sediment median particle size. $g(s-1)$ is an independent quantity defined from $s=\rho_{s} / \rho$ and gravitational acceleration, $g$, where $\rho$ and $\rho_{s}$ are the fluid and sediment 
(particle) densities, respectively. Finally, although particle fall velocity $\left(w_{s}\right)$ may be derived from the other parameters if the particle shape is known, it has also been included in Eq. 2 as an independent parameter for generality.

Noting that there are three primary dimensions, Eq. 2 can be rewritten as:

$$
S_{0} / D=f\left(K C=\frac{2 \pi A}{D}, \beta=\frac{D^{2}}{T \nu}, \frac{h_{\text {min }}}{D}, \psi=\frac{(2 \pi A / T)^{2}}{g(s-1) d_{50}}, \frac{2 \pi A / T}{w_{s}}\right)
$$

The first dimensionless group is $K C$ number, the second is the ratio of $R e$ to $K C$ number (commonly referred to as $\beta$ ), the third is the minimum gap ratio, the fourth describes a sediment mobility number $\psi$ written in terms of the maximum cylinder oscillation velocity $\left(V_{\max }=2 \pi A / T\right)$ and the final group is the ratio of maximum cylinder velocity to fall velocity (somewhat analogous to the Dean Number; Hughes, 1993). Across the first four independent groups, the present experimental program covers a realistic range for a single sand sediment. Although the final group is not explored systematically in this work, it is included for future reference since mode of erosion is known to affect trench geometry and rate of development (e.g. Mohr et al., 2016).

At full scale, the relevant motion of risers, pipelines or cables depends on aspects such as water depth, system configuration, system geometry and structural properties, operating metocean conditions, the response of an attached vessel and the geotechnical response of the seabed (e.g. for pipe installation or laying, see Westgate et al., 2010a). Limited published data in the literature exists regarding actual motions at the seabed, but inferences may be made to form a representative parameter range for testing. For example:

1. Full scale riser tests conducted during the STRIDE JIP (Bridge, 2005) investigated vertical motions of a $0.168 \mathrm{~m}$ diameter riser corresponding (at $10 \mathrm{~m}$ above seabed) to a $[K C, T]$ of $[1.9,6 \mathrm{~s}]$ and $[15$, $25 \mathrm{~s}$ ] for operating and extreme motions of the attached floating system, respectively. These motions were adopted based on riser analyses conducted as part of the JIP.

2. Westgate et al. (2010b) reported video footage of $0.32 \mathrm{~m}$ diameter pipeline motions during the lay process of up to $K C=5.0$ in the near-bed region with an average wave period of $5 \mathrm{~s}$. That pipeline had a recorded trench depth (where sediment was suggested to have been eroded) in some locations of 1 to 1.5 diameters.

3. Bridge and Howells (2007) report riser trenches in the field ranging from 2 to 4.5 diameter deep; this suggests the $K C$ number may reach up to 6 to 14 if it is assumed the riser returns to the original seabed level and contacts the bed in a cycle.

4. Geotechnical centrifuge testing undertaken by Elliott et al. (2013) considered a maximum $K C$ value of about 14 and a period $T$ of $20 \mathrm{~s}$ (but also $1 \mathrm{~s}$, in an attempt to scale the motions for centrifuge modelling) defined at prototype scale for a point on the riser $5 \mathrm{~m}$ above the seabed. The riser had a 
prototype diameter of $0.508 \mathrm{~m}$.

5. Hejazi (2018) provided the authors with example motions from dynamic riser analyses for a $0.228 \mathrm{~m}$ diameter riser in 1,000 $\mathrm{m}$ water depth for typical operational sea states corresponding to $0.4 \mathrm{~m}$ vertical amplitude motions of a semi-submersible host. The motions for this specific example in the touchdown zone resulted in a $K C$ number of about 6.9 and an oscillation period $T$ of $13 \mathrm{~s}$.

From these references it appears that $K C<15$ is broadly representative of pipeline and riser motions induced by a floating host near the touchdown zone. For pipelines being installed, the range is likely to be smaller (i.e. $K C<5$ ) since pipelay occurs in relatively calm conditions. The range in quoted oscillation period suggests $R e \sim \mathcal{O}\left(10^{4}-10^{5}\right)$ or $\beta \sim \mathcal{O}\left(10^{3}-10^{4}\right)$.

For the present study, two series of tests were conducted: the first (main) series focused on observations and measurements of trench formation, whilst the second (supplementary) series focused on the threshold of sediment motion. The experiments that were performed in the first series are listed in Table 2. These experiments were chosen to cover a representative range in $K C$ number and $\beta$ number. The mobility number was varied whilst fixing the minimum gap ratio at a reference value of approximately 0.1 to ensure a range of near bed velocities.

For the second supplementary series, $K C$ was varied up to 12 and $\psi$ was varied so as to achieve sediment motion. Three different pipe diameters were adopted to vary $\beta$ between $\sim \mathcal{O}\left(10^{2}-10^{3}\right)$, and the minimum gap height was varied between 5 to $20 \%$ of the cylinder diameter.

\section{Results: General Observations}

A range of different trench geometries are observed across the parameter space investigated in the main experiments. Figure 2 shows a selection of seabed cross-sections recorded during the experiments. Figure 3 shows the corresponding centreline bed level progression over time. General observations can be made as follows:

1. Sediment transport leading to trench formation is generally confined to the seabed local to the cylinder, with the width of the seabed affected by sediment transport generally increasing in size with increases in $K C$ number and $\psi$, up to $\pm 2 D$.

2. Trench formation coincides with the formation of berms above the initial bed level, with similar crosssectional area to the trench indicating that the sediment moves primarily in bed load or, if suspended, it is deposited locally.

3. Trenching occurs quickly in the early stages of cycling and continues in most cases at a reducing rate towards an equilibrium geometry. These findings suggest that transport patterns are linked to the 


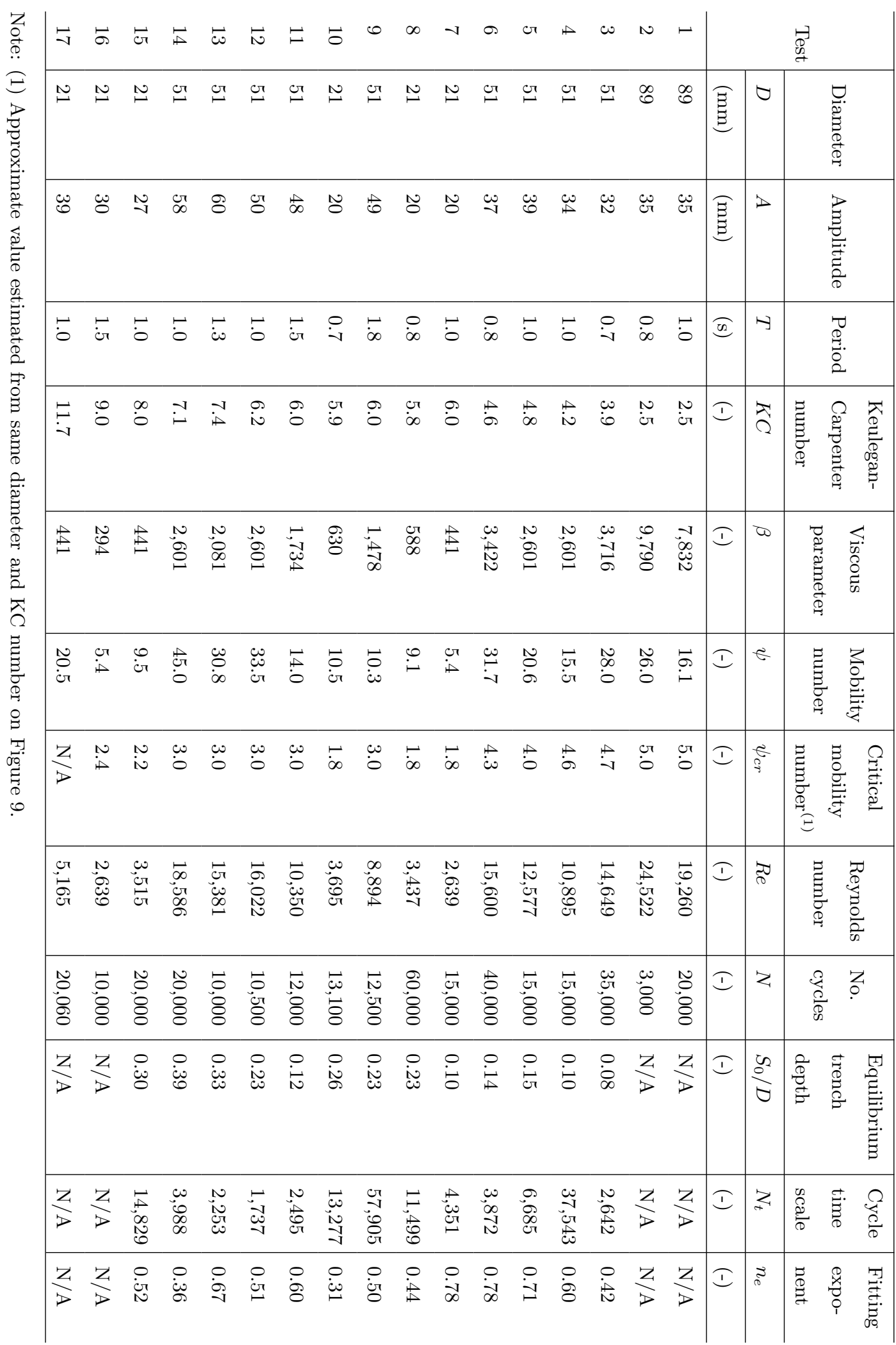


scale of the fluid flow induced by cylinder oscillations and depend significantly on the motion of the cylinder.

In addition to these general observations, there are noticeable differences in both the trench profiles and how the bed evolves with time. These changes are primarily dependent on $K C$ number since this parameter primarily controls changes in the overall vortex shedding dynamics (see Section 1). Three primary regimes of trench formation have been identified based on the main experimental results. These are described in further detail in the following sections.

\subsection{Symmetric central ridge $(K C<4)$}

For $K C<4$ a central ridge of sediment forms beneath the centreline of the cylinder, together with two trenches on either side (see Figure 2a, b). At the commencement of each experiment, sediment motion occurs primarily via bedload transport when the cylinder is close to the bed (i.e. $0.75<t / T<1.2$ ). This sediment motion is directed away from the centreline when the cylinder approaches the bed and towards the centreline as the cylinder moves upward. The transport is symmetric about the cylinder centreline but is not time-symmetric over a cycle. This results in net transport towards the centre of the cylinder. Over multiple oscillations this net transport leads to formation of a ridge (see Figure 4a).

To illustrate these observations, Figure 4b-e provides sketches of the sediment transport observed at different instances during a cycle. Also shown on this figure (in grey lines) are the flow features identified via flow visualisation experiments for a cylinder oscillating with similar $K C$ number above a rigid wall (Tom, 2018). The main flow features include the vortex dynamics and pumping of water into and out of the gap beneath the cylinder. For this regime, these flow features are consistent with the observed sediment transport mechanisms. In particular, symmetric sediment motion either side of the cylinder is consistent with pumping flow beneath the cylinder, which is symmetric and peaks at a similar phase during the oscillation (Tom, 2018). In addition, the time period over which sediment motion occurs during the second half of the cycle (i.e. cylinder moving towards the bed) was slightly longer than the first half-cycle. This difference is attributed to the background circulation in the flow field directed outward near the bed (indicated by the circulation cells on Figure 4b-e), which prolongs transport as the cylinder moves towards the bed.

\subsection{Symmetric trench with intermittently asymmetric transport $(4<K C<9)$}

For the experiments where $4<K C<9$, the eventual trench geometries in Figure 2 is symmetric about the cylinder centreline, with generally no prominent ridge above the initial bed level. However, although the final geometries are qualitatively similar throughout this regime, the dominant mechanisms of sediment transport observed during individual cycles varies.

For $4<K C<6$, bedload transport similar to that described in the previous subsection still contributes significantly to sediment movement over the time interval $t / T=0.75$ to 1.2 . However, sediment movement 


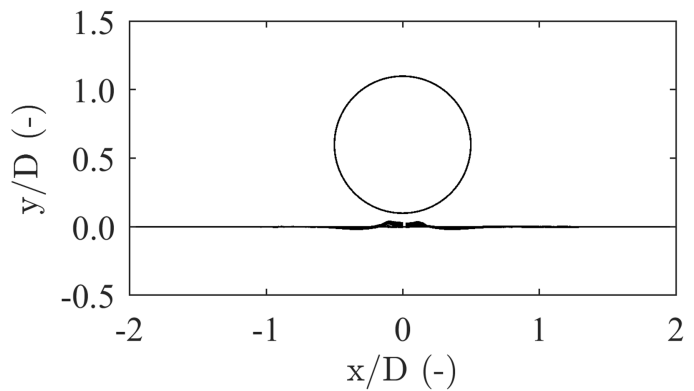

(a) Test $1, K C=2.5 ; \psi=16.1$

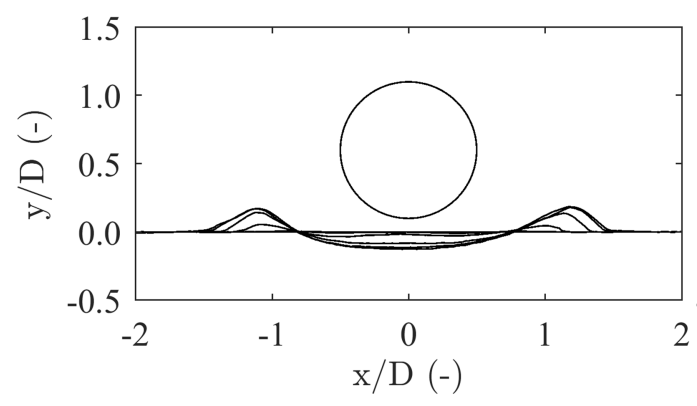

(c) Test $5, K C=4.8 ; \psi=20.6$

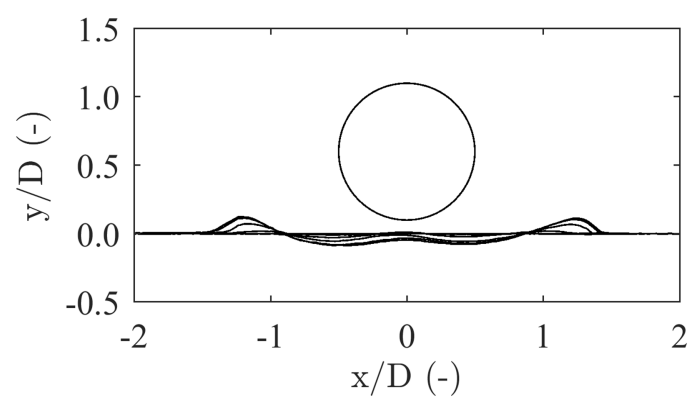

(e) Test $9, K C=6.0 ; \psi=10.3$

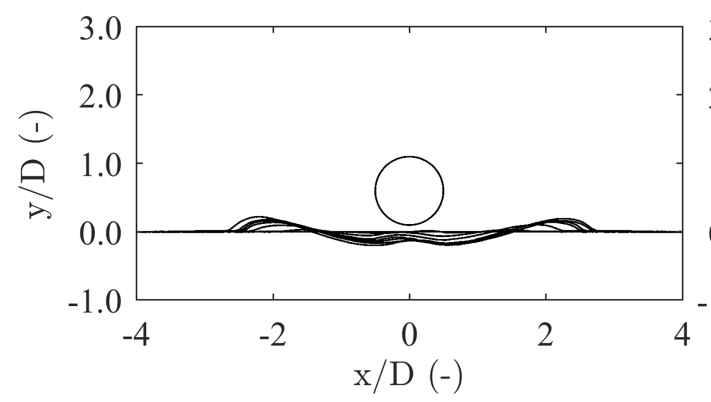

(g) Test $15, K C=8.0 ; \psi=9.5$

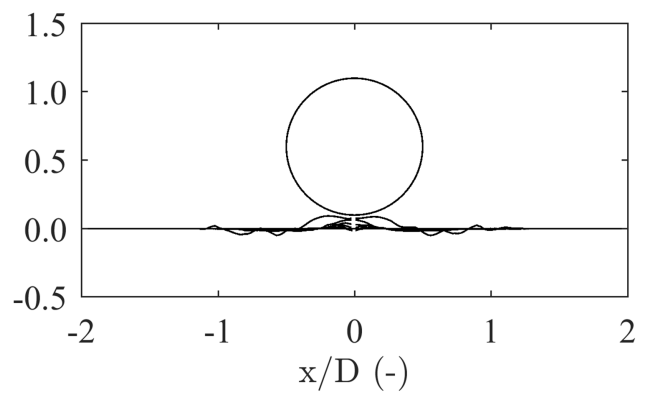

(b) Test $2, K C=2.5 ; \psi=26.0$

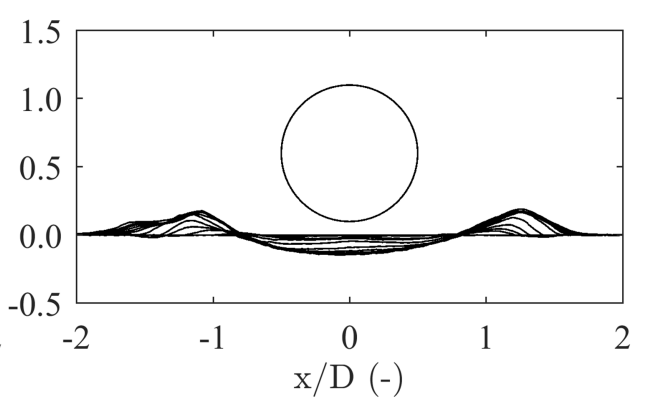

(d) Test $6, K C=4.6 ; \psi=31.7$

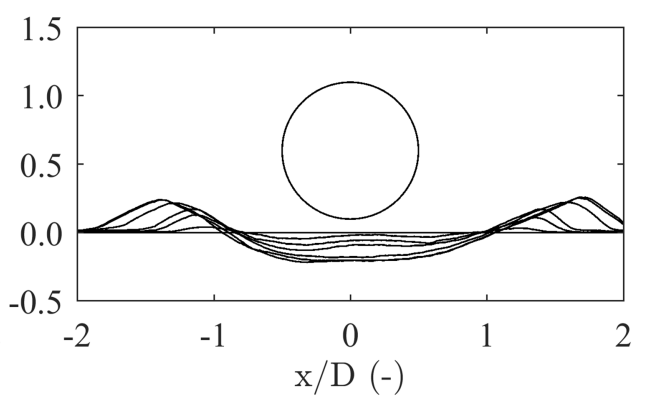

(f) Test $12, K C=6.2 ; \psi=33.5$

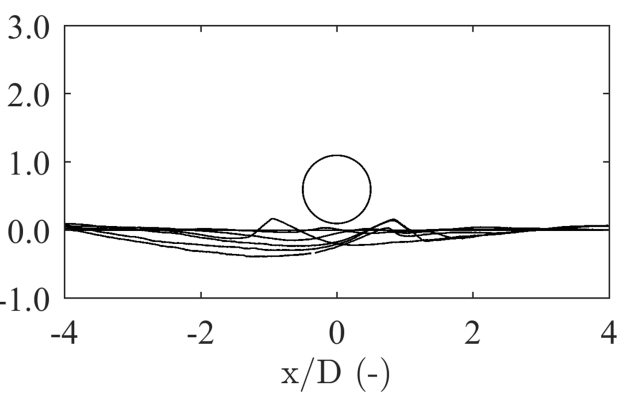

(h) Test $17, K C=11.7 ; \psi=20.5$

Fig. 2. Measured trench profiles after progressively increasing numbers of cycles. Cylinder represents lowest position in cycle. 


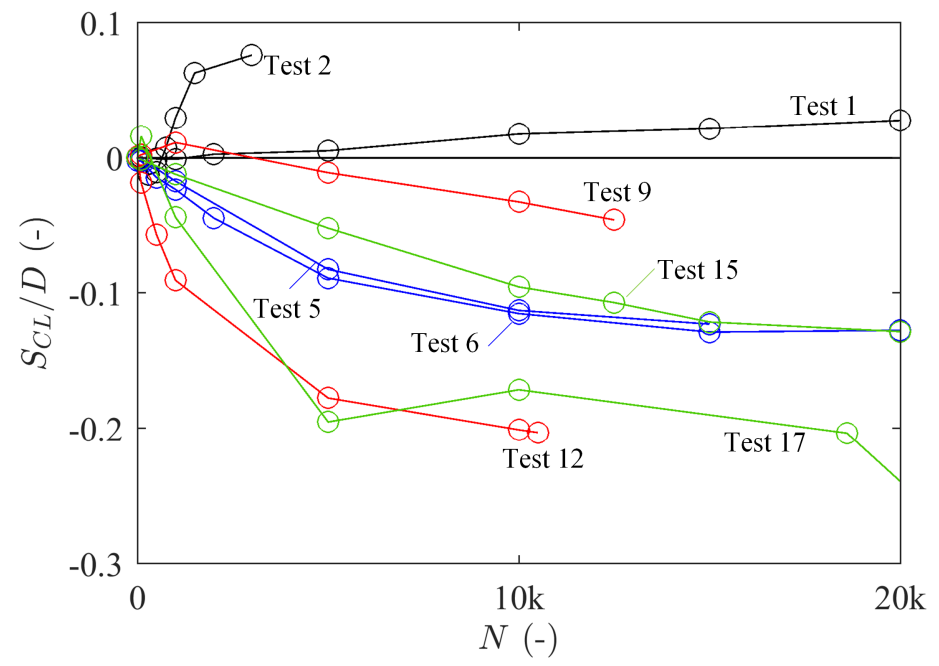

Fig. 3. Measured trench depth progression at the centre of the cylinder (i.e. $S_{C L}=\xi_{0}-\xi(0,0, t)$ ). Lines grouped by colour for similar $K C$ numbers.

also occurs due to spontaneous localised transport events in which sediment is temporarily suspended within a localised area (e.g. Figure 5a). These localised events occur on either side of the cylinder, although not generally on both sides at the same position along the cylinder during a cycle. The events occur at a slightly different time interval in the cycle compared to the bedload transport $(0.1<t / T<0.5$, as the cylinder moves away from the bed). For some cycles, the regions of sediment associated with the localised events are sufficiently large to encompass sediment at the centreline of the cylinder, which appears to inhibit ridge formation. With increasing $K C$ number and $\psi$, the contribution to total sediment transport due to the localised events appears to increase relative to that due to bedload transport.

To summarise these observations for $4<K C<6$, Figure 5b-e presents sketches similar to Figure 4 , which also include flow features simplified from Tom (2018) for a similar $K C$ number range. These flow features indicate the formation of asymmetric trailing vortices during each half cycle, which detach when the cylinder reverses direction. When the larger of these detached vortices sheds at the lowest point of the cylinder motion (i.e. Vortex A on Figure $5 \mathrm{c}$ and $5 \mathrm{~d}$ ), it advects back towards the bed and induces a jet-like flow between itself and the newly forming vortex on the same side of the cylinder (Vortex $\mathrm{C}$ on Figure 5d). At around $t / T \sim 0.1$, the jet-like flow impinges on the bed and is believed to cause the localised sediment suspension events.

In terms of net sediment transport, the asymmetry in the vortex shedding shown on Figure 5 was observed to switch frequently in Tom (2018), such that jet flow often occurs on either side of the cylinder on different cycles. This switching is consistent with the observation of localised transport events occurring on both sides of the cylinder but not at the same position. This frequent switching is believed to be the reason 


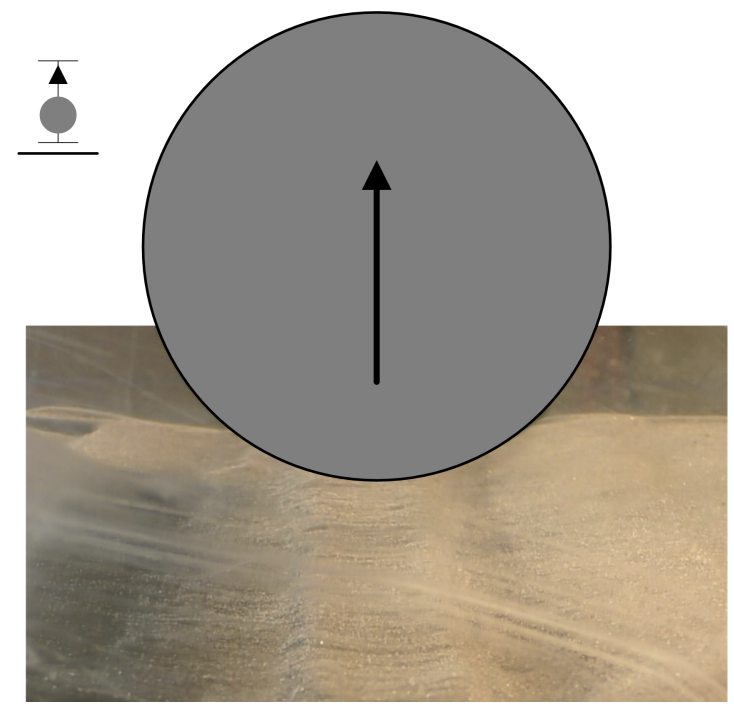

(a) Example video still from $K C=3.6, \psi=17.6$ after 2000 cycles.
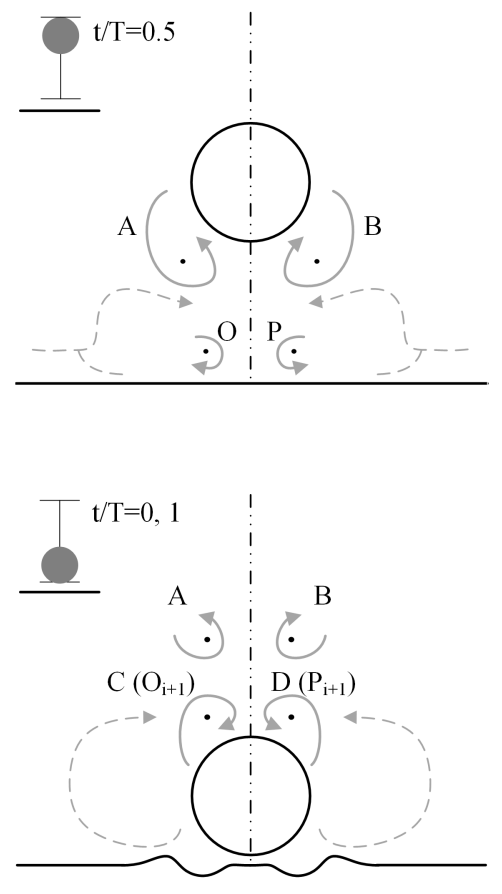

No pumping flow

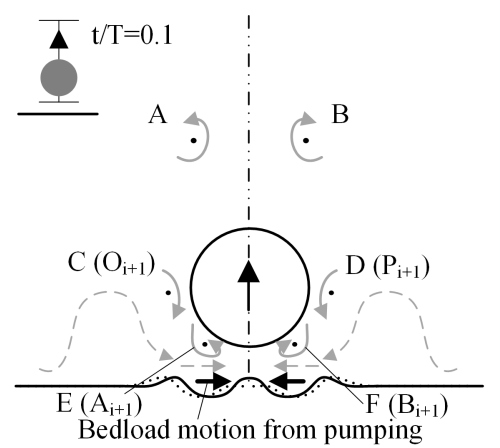

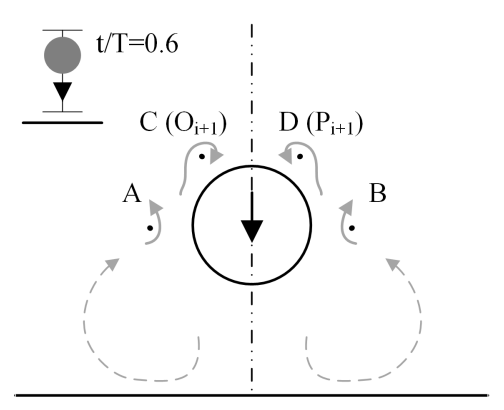

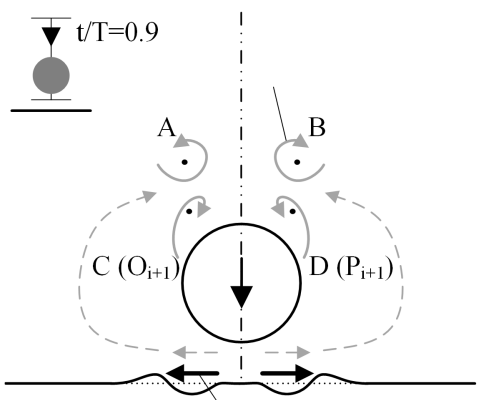

Bedload motion from pumping

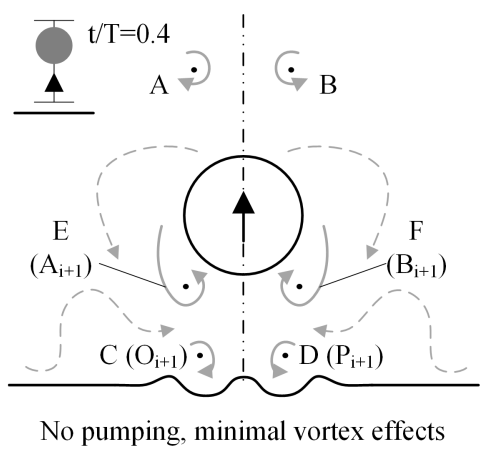

(b) Idealised flow and transport schematic

Fig. 4. Regime 1 - Example video still and idealised representation of flow dynamics and seabed formation. Solid grey lines represent vortices with cores denoted by dots. Dashed grey lines represent pumping or overall circulation features. 
why the final trench geometry is approximately symmetric.

For larger $K C$ numbers in this regime (i.e. $6<K C<9$ ), localised transport events are also observed; but a key additional observation is the occurrence at $t / T \sim 0,1$ of uni-directional (i.e. asymmetric) bedload transport across the cylinder axis on some (but not every) cycle. This asymmetric transport removes sediment from the centre of the cylinder. The direction of bedload transport is usually away from regions with localised transport, and appears to switch direction intermittently over 10 s of cycles. This intermittency is consistent with results reported by Tom (2018) suggesting that for $5 \leq K C<8, \beta=500$ the side on which the dominant trailing vortex forms and the direction of near-wall horizontal flow at the cylinder centreline varies between individual cycles. Analogously, Tatsuno and Bearman (1990) described a flow regime (Regime E) wherein the direction of asymmetric vortex behaviour periodically switches sides. This was linked to three-dimensional flow structures moving irregularly along the cylinder in time. The general observations in this study for $6<K C<9$ are conceptually similar to those illustrated on Figure 4b-e, except that the bedload transport becomes more asymmetric beneath the cylinder.

\subsection{Asymmetric trench $(9<K C<12)$}

For $K C>9$, the trench geometry is asymmetric throughout much of its development. To quantify this shift from symmetric to asymmetric geometry, Figure 6 shows the horizontal location of the deepest point in the trench on the left and right hand sides of the cylinder (averaged along the cylinder length). Results are shown for all tests for which $>1000$ cycles were completed (some of which are not detailed in Table 2 ). For a symmetric trench, $\left|x_{\max , R S}+x_{\max , L S}\right|=0$, so the vertical axis in this figure would be zero. Since $x_{\max , R S} \geq 0$ and $x_{\max , L S} \leq 0$, large values of $\left|x_{\max , R S}+x_{\max , L S}\right|$ indicates that the deepest position on one side of the cylinder is further from the centreline than the deepest position on the other side. For tests conducted at $K C \lesssim 9$, the trench profiles measured are approximately symmetric after many cycles. In contrast, for $K C \gtrsim 9,\left|x_{\max , R S}+x_{\max , L S}\right|$ is often larger than a cylinder diameter, indicating large asymmetry in the trench profile.

The general mechanisms of sediment transport observed in this asymmetric regime are similar to that observed for $6<K C<9$ and comprise asymmetric bedload transport and localised transport events. However, for $K C>9$ the sediment motion during the initial cycles (both the direction of bedload transport and the location of localised events) are much more uniform along the cylinder (see Figure 7a) and maintain the same sense of asymmetry for tens to hundreds of cycles without switching. This observation is consistent with Tom (2018), who showed that over this $K C$ range consistent vortex shedding in a single primary direction was also maintained over $\mathcal{O}(10)$ to $\mathcal{O}(100)$ of cycles coincident with the development of a transverse vortex street.

To explain the observed trends in the sediment motion, Figure 7b-e again documents the flow field and sediment motion during a cycle. This flow field is similar to that shown on Figure 5, except that the dominant 


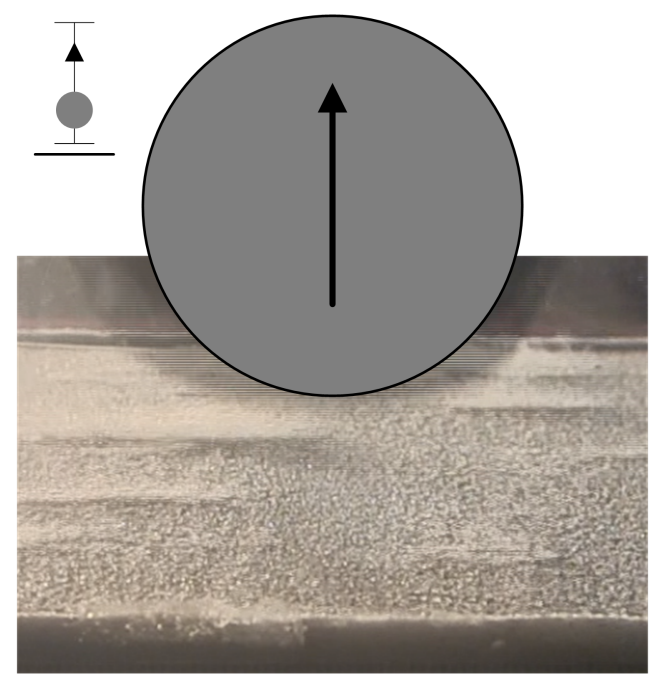

(a) Example video still from $K C=6.1, \psi=$ 33.5 after 1 cycle.
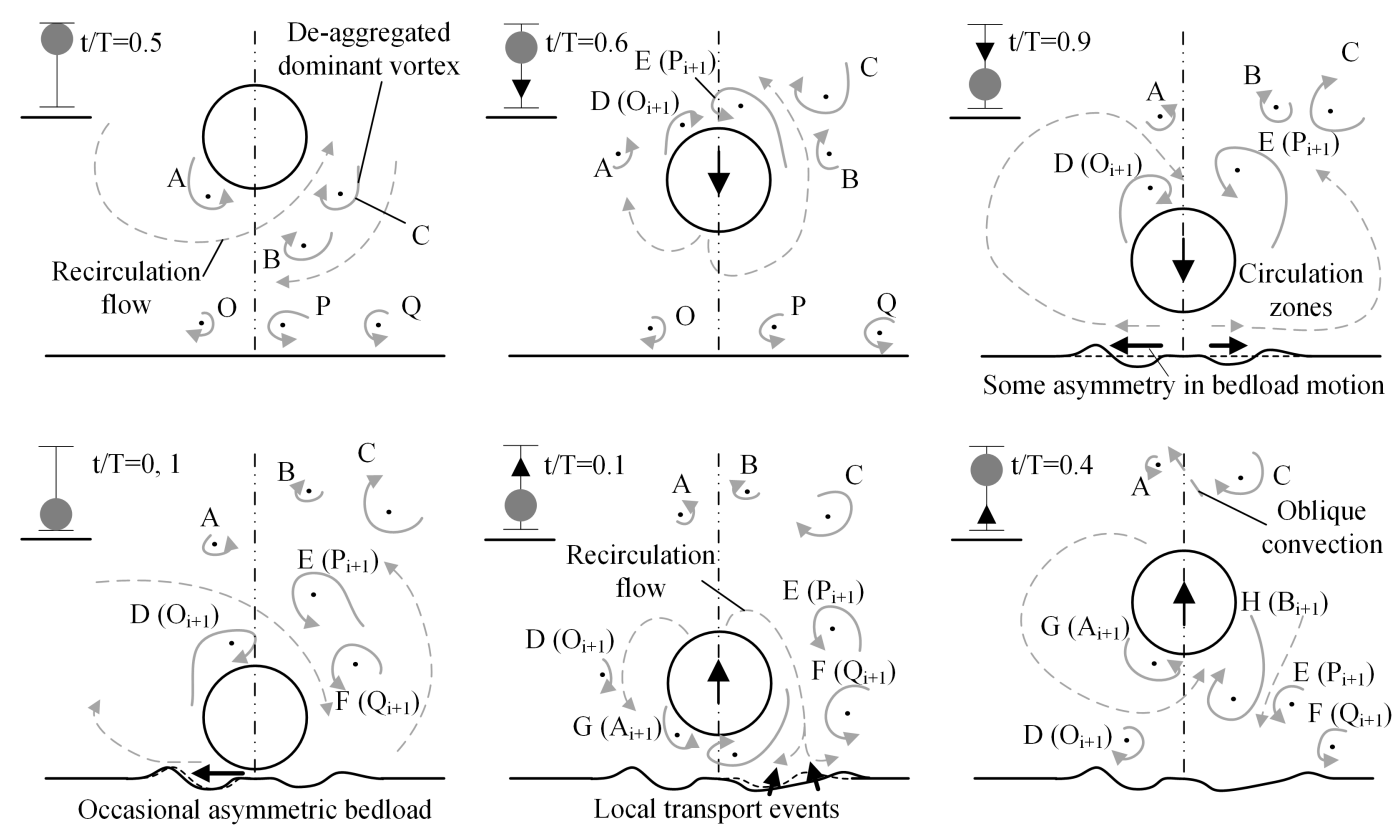

(b) Idealised flow and transport schematic

Fig. 5. Regime 2 - Example video still and idealised representation of flow dynamics and seabed formation. Solid grey lines represent vortices with cores denoted by dots. Dashed grey lines represent pumping or overall circulation features. 


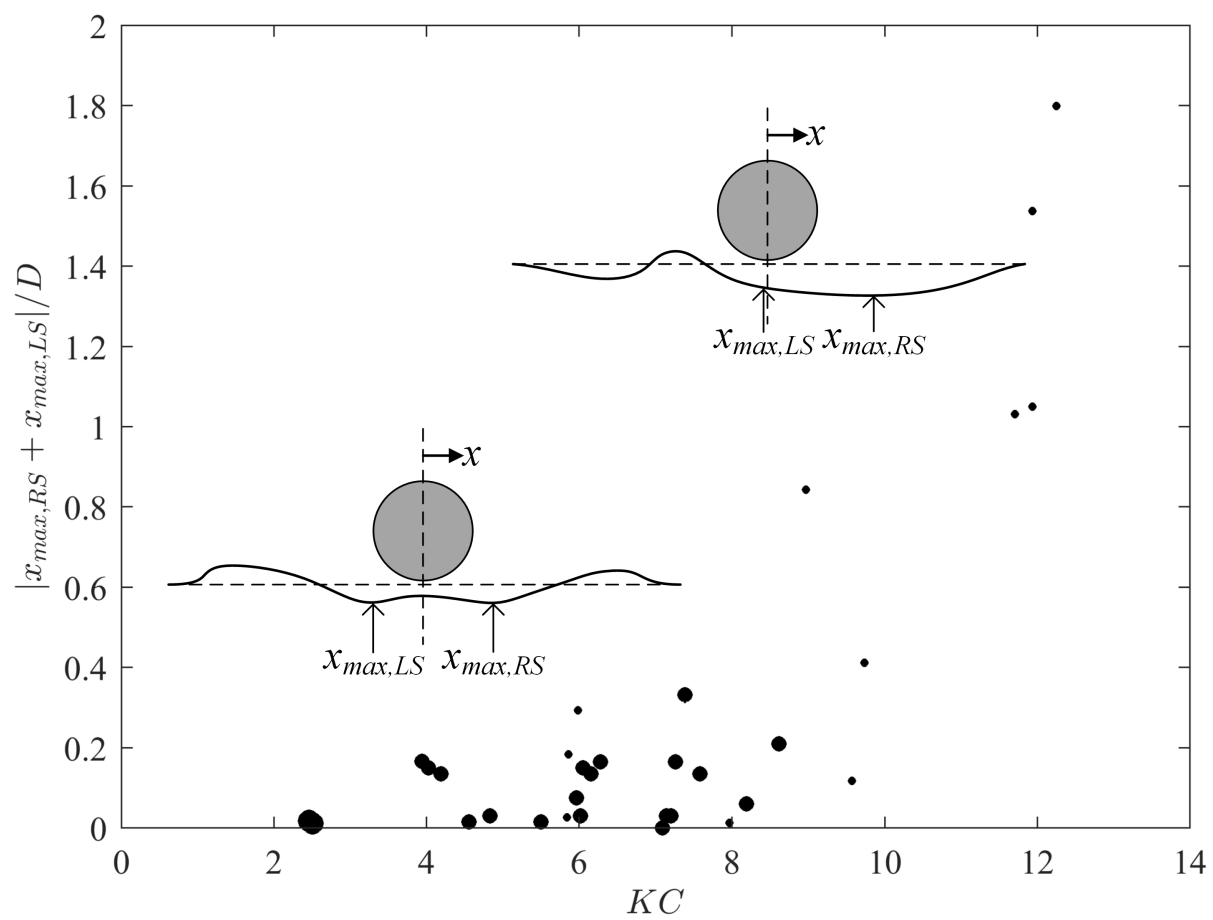

Fig. 6. Normalised distance between locations of maximum trench depth on right and left sides of cylinder. Data for all tests with $N>1,000$.

trailing vortex (labelled Vortex A on Figure 7c) now detaches prior to the cylinder reversing direction. This results in the formation of a transverse vortex street. However, the jet-like flow that forms between this detached vortex and the newly forming trailing vortex (Vortex B on Figure 7d, e) is still evident and causes localised transport. The asymmetric flow near the bed at $t / T \sim 0$ (Figure $7 \mathrm{c}$ ) coincides with the overall circulation dynamics observed by Tom (2018) for $K C>8$. This asymmetry is similar to but more consistent over multiple cycles than that described for Regime 2.

\subsection{Uniformity of trench geometry}

The preceding results focused on only $2 \mathrm{D}$ features of sediment transport and the flow field. For low to moderate $K C$ numbers (i.e. $K C<9$ ) these features dominate, with uniform trenches forming along the cylinder length throughout the trenching process (see Figure 8). In contrast, for $K C>9$ the trenches are more non-uniform along their length and appear to be affected by the end conditions, even for tests with $L / D=30$. Figure 8d, for example, shows the trench width and depth varying along the cylinder axis. Increased transport also occurs near the ends of the cylinder, suggesting end-effects; and for large $K C$ the sediment motion is clearly three-dimensional. Quantitative results presented in this paper for $K C>9$ should therefore be treated cautiously. However, the qualitative discussion of the trenching mechanics seen 


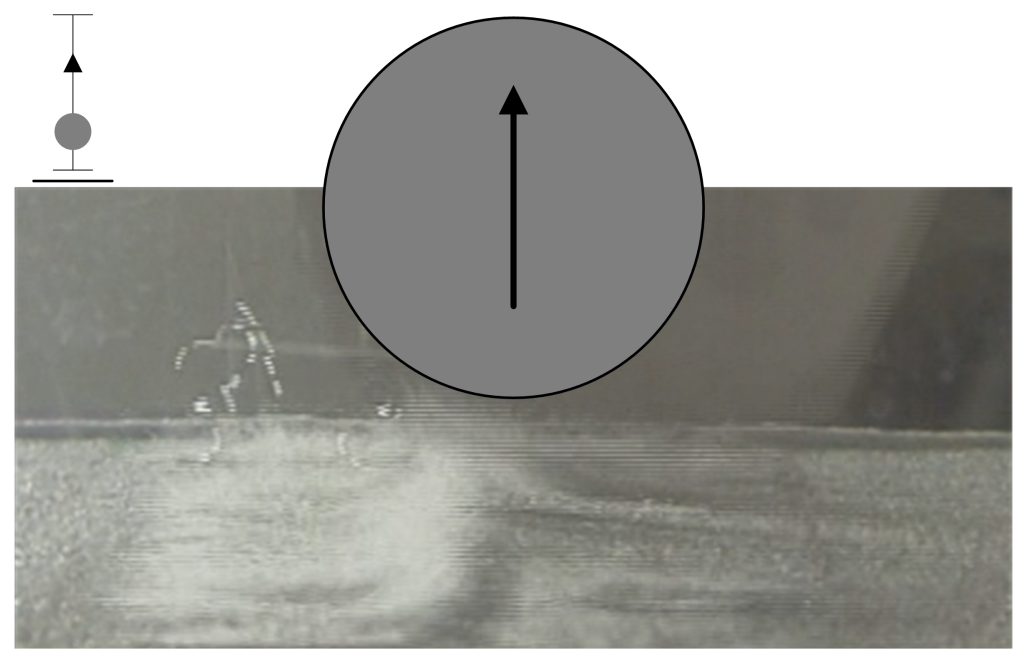

(a) Example video still from $K C=12.3, \psi=33.4$ after 1 cycle. (not listed in Table 2)
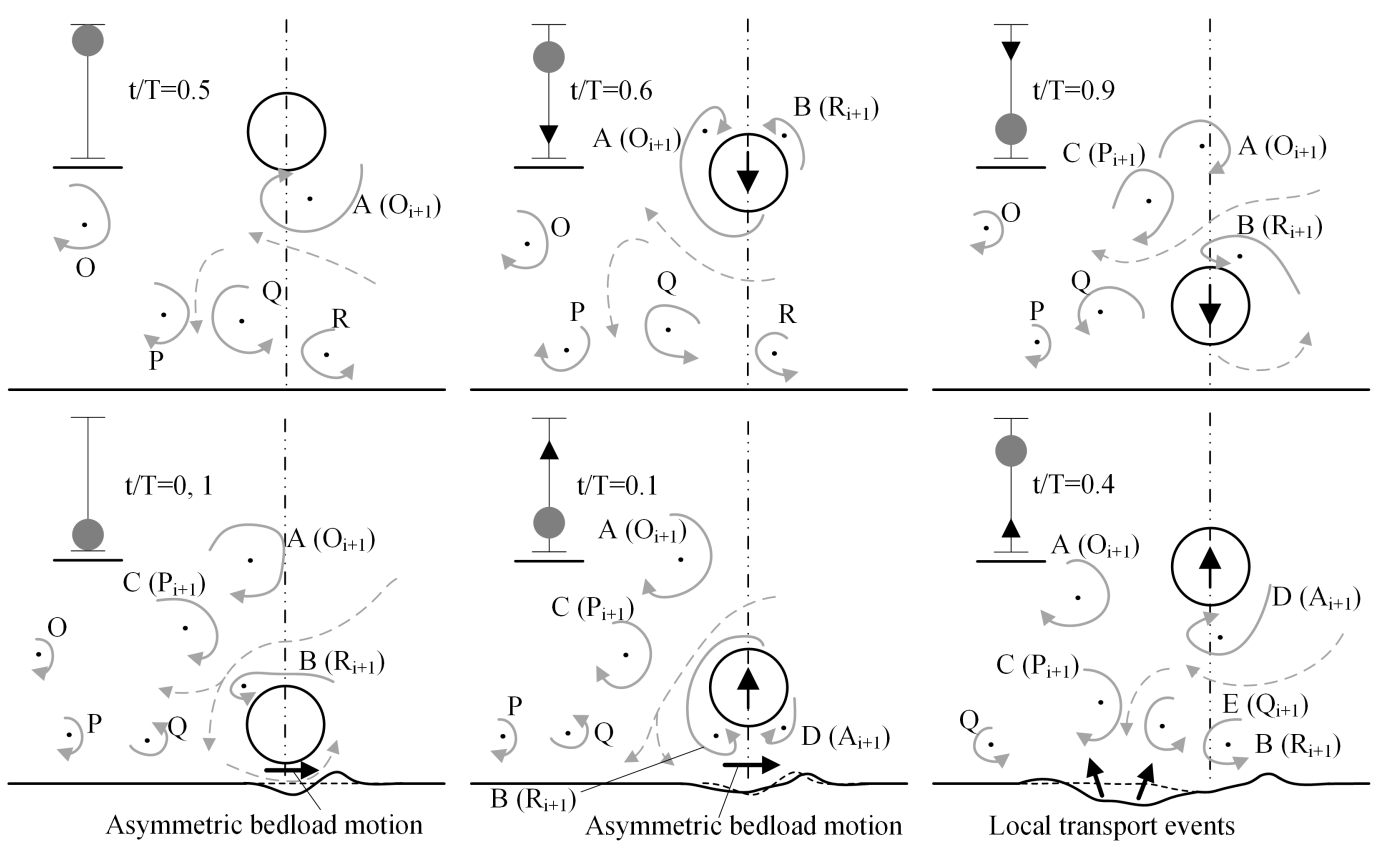

(b) Idealised flow and transport schematic

Fig. 7. Regime 3 - Example video still and idealised representation of flow dynamics and seabed formation. Solid grey lines represent vortices with cores denoted by dots. Dashed grey lines represent pumping or overall circulation features. 


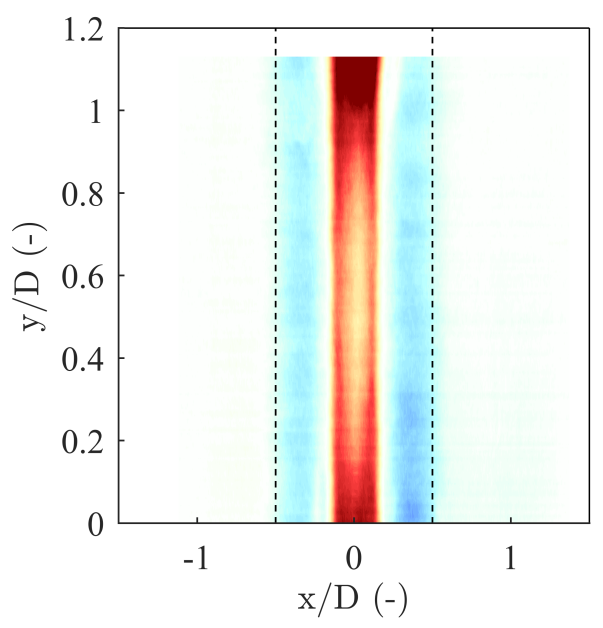

(a) $K C=2.5, \psi=16.1$, Range $\pm 0.05 D$

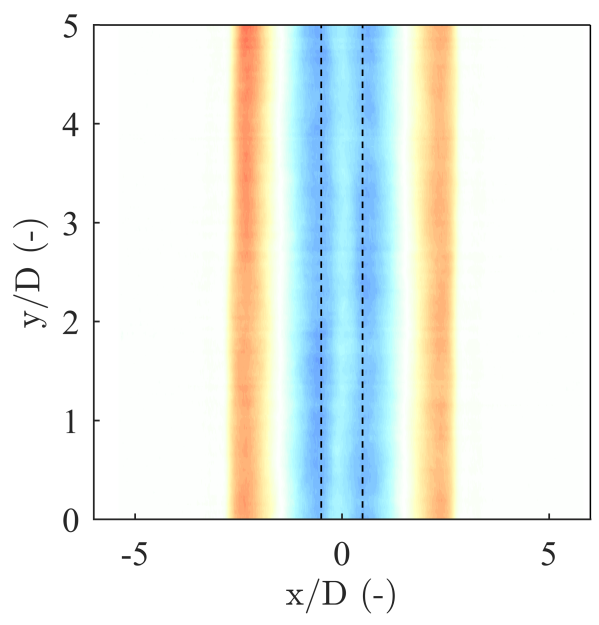

(c) $K C=9.5, \psi=9.5$, Range $\pm 0.05 D$

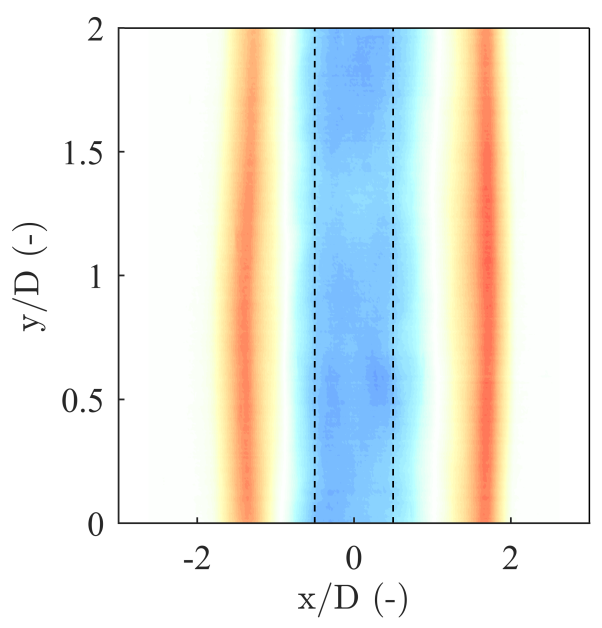

(b) $K C=6.1, \psi=33.5$, Range $\pm 0.05 D$

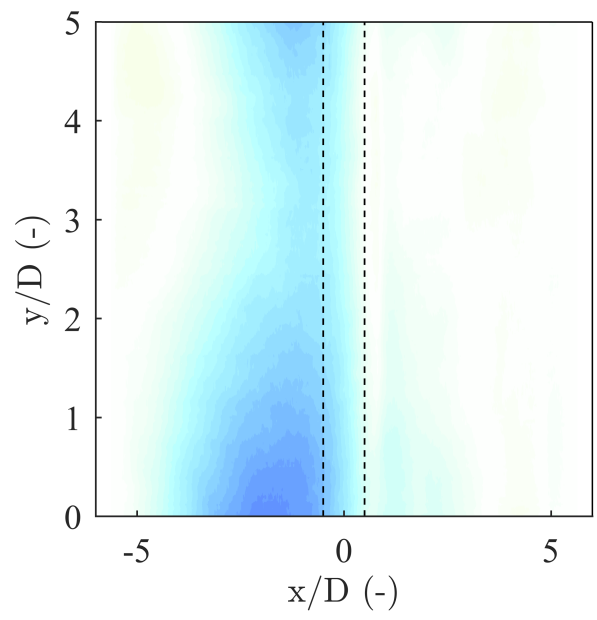

(d) $K C=12.3, \psi=20.5$, Range $\pm 0.05 D$

Fig. 8. Final surface scan elevation over middle $100 \mathrm{~mm}$ of cylinder length. Depth values normalised by cylinder diameter. Colour range noted in subcaptions.

at the mid-plane of the flume is representative of this $K C$ number regime regardless.

\section{Quantitative results: trench formation}

\subsection{Threshold of sediment motion}

To predict trenching, the conditions under which sediment motion occurs must first be assessed. Experiments were undertaken to assess the cylinder motions for which sediment was observed to be mobilised. 
cylinder above an initially flat bed for approximately 100 cycles at a constant value of $\psi$. This process was then repeated for increasing $\psi$. During these oscillations, sediment motion was defined as occurring when one of two observations were made:

1. Method 1: grains were transported in bedload a distance exceeding one grain diameter during the cycle over more than $50 \%$ of the cylinder length; or

2. Method 2: sediment was mobilised (in bedload or suspension) due to localised transport events at least once per cycle over more than 10 cycles.

Mobility via the first observation was most prevalent at low $K C$ numbers. The second observation was more common at higher $K C$ numbers where localised transport events are more prevalent. For a given value of $K C$ number, the smallest value of $\psi$ that corresponds to occurrence of sediment motion (i.e. the critical mobility number) is denoted as $\psi_{c r}$ throughout the remainder of this paper.

Figure 9 summarises the threshold of motion observations in terms of $K C$ number and $\psi$ for different diameters and values of $h_{\min } / D$. In all cases for $K C<4$ and approximately constant $h_{\min } / D$, the required $\psi$ for sediment motion increases with $K C$. This implies that for this range in $K C$ number, as amplitude of motion increases, the cylinder must move faster to achieve a near-bed fluid velocity sufficient to mobilise the sediment. The results also indicate that in the range of $\psi$ near the sediment mobility threshold the transport regime primarily involves symmetric bedload transport for $K C<4$.

In contrast, for $K C \gtrsim 4$ the required $\psi$ for sediment motion reduces slightly for $4<K C<6$ (at least for $D=51 \mathrm{~mm}$ ) and then changes only slightly for $K C>6$. This reduction suggests that as $K C$ increases there is an apparent amplification in the near-bed velocities, which enables sediment to be mobilised at relatively lower $\psi$. Initiation of sediment motion in this regime also coincides with the occurrence of localised transport events described previously.

Aside from the trends with $K C$ number, Figures 9c, d and e also indicate that for $K C<4$ the cylinder motions required for mobility are dependent on the minimum distance between the cylinder and the seabed. When the minimum gap is larger, the rate of volume change in the gap beneath the cylinder decreases. Thus, faster cylinder motions are required to cause the same near bed fluid velocities at the same cycle phase. At higher $K C$ numbers (see Figure 9b), this dependency is less clear because the sediment motion is caused by localised events.

\subsubsection{Prediction of threshold of motion at low $K C$ number}

Provided the $K C$ number is small (i.e. $K C \lesssim 4$ ) and $h_{\min } / D$ is small, Tom (2018) showed that continuity arguments can be used to estimate the flow velocity beneath the cylinder. This is possible because small $K C$ ensures the flow is symmetric and free from significant vortex shedding influence. Additionally, small $h_{\text {min }} / D$ ensures the flow is relatively uniform with height in the gap between the cylinder and wall, such 


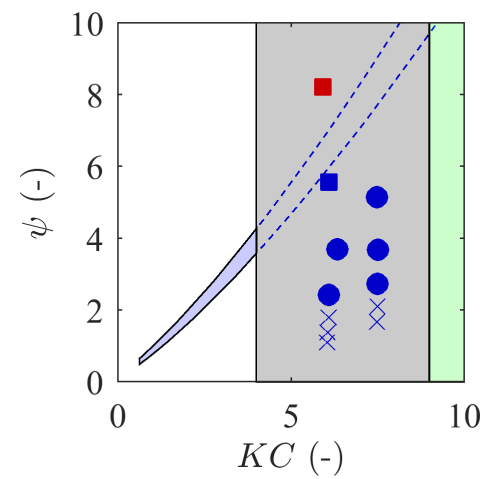

(a) $D=21 \mathrm{~mm}$

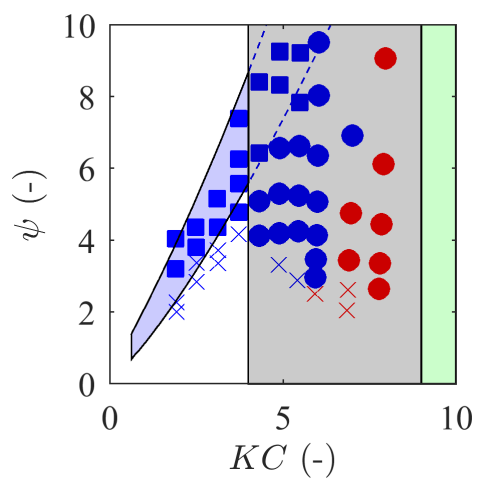

(b) $D=51 \mathrm{~mm}$

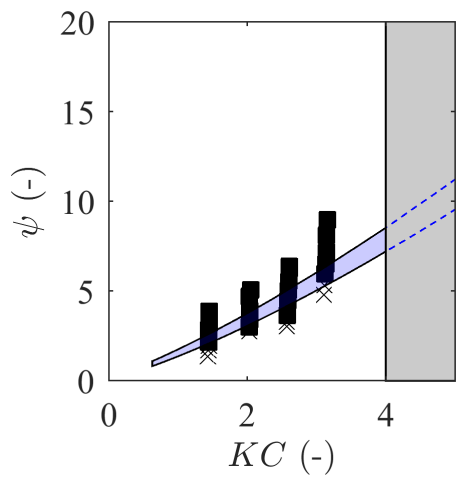

(c) $D=89 \mathrm{~mm}, h_{\min } / D<0.07$

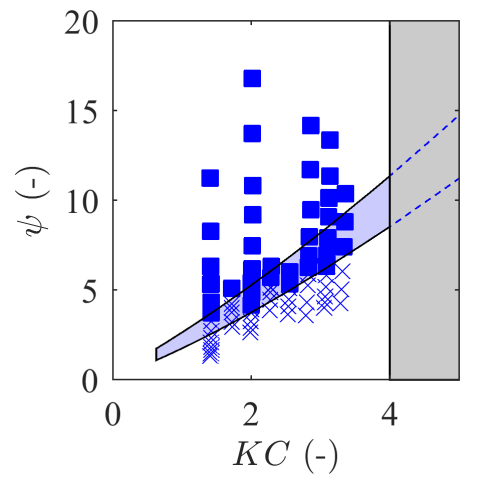

(d) $D=89 \mathrm{~mm}, 0.07 \leq h_{\min } / D<$ 0.14

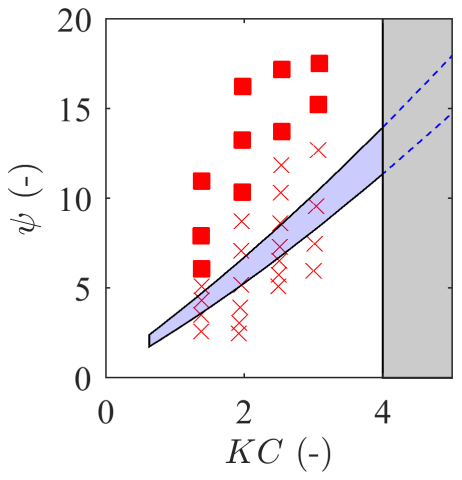

(e) $D=89 \mathrm{~mm}, h_{\min } / D \geq 0.14$

Fig. 9. Threshold of motion. Squares - Method 1. Circles - Method 2. Solid symbols - observed sediment mobility. $\times$ - no motion. Black symbols $-h_{\min } / D<0.07$. Blue symbols $-0.07<h_{\min } / D<0.14$. Red symbols $h_{\text {min }} / D>0.14$. Shaded areas - approximate ranges for regimes in Section 4. Blue shaded areas - predictions based on Eq. 7 to 13 for measured range in $h_{m i n} / D$. 


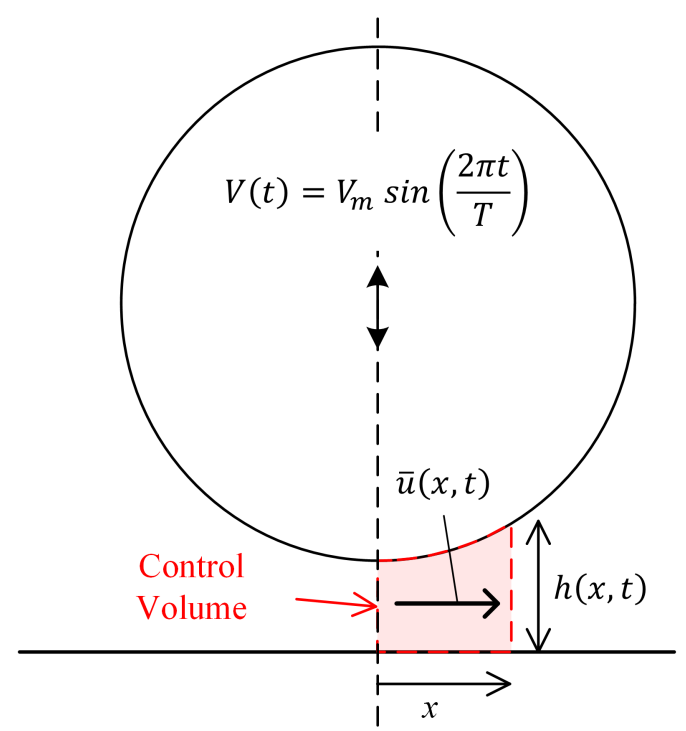

Fig. 10. Control volume between an oscillating cylinder and a rigid wall.

that the depth-averaged velocity deduced from a control volume analysis is representative of that at any height within the gap. In this subsection, such estimates of flow velocity are used to predict the threshold of motion beneath the cylinder.

To obtain an expression for the velocity beneath the cylinder using continuity arguments, a control volume can be introduced (dashed region on Figure 10). The change in size of the control volume during an oscillation can be equated to the flow passing through its boundary. Due to symmetry only the right-hand boundary in Figure 10 allows flow to pass, and hence continuity is satisfied if

$$
\frac{\partial \Omega(x, t)}{\partial t}=\bar{u}(x, t) h(x, t)
$$

where $\Omega(x, t)$ represents the volume (per unit length) of the control volume, $h(x, t)$ is the height at the right-hand boundary of the control volume and $\bar{u}(x, t)$ is the depth-averaged horizontal velocity moving across the boundary of the control volume.

Given that the cylinder is moving sinusoidally in time, it follows that

$$
\frac{\partial \Omega(x, t)}{\partial t}=x \frac{2 \pi A}{T} \sin \left(\frac{2 \pi t}{T}\right)
$$

and

$$
h(x, t)=h_{\min }+A-A \cos \left(\frac{2 \pi t}{T}\right)-\frac{D}{2}\left(1-\left(\frac{2 x}{D}\right)^{2}\right)^{0.5} \text { for } x \leq \frac{D}{2}
$$

Substituting Eq. 5 and 6 into 4 gives, after some rearrangement,

$$
\frac{\bar{u}(x, t)}{V_{\max }}=\frac{\frac{x}{A} \sin \left(\frac{2 \pi t}{T}\right)}{\frac{h_{\min }}{A}+\left(1-\cos \left(\frac{2 \pi t}{T}\right)\right)+\frac{\pi}{K C}\left[1-\left(1-\left(\frac{2 x}{D}\right)^{2}\right)^{0.5}\right]}
$$


where $V_{\max }$ is the maximum cylinder velocity. Although the form of Eq. 7 is complicated, it illustrates several key points. Firstly, when the minimum gap between the cylinder and the bed is small relative to the amplitude of motion (i.e. $h_{\min } / A<<1$ ), the pumping velocity increases. Secondly, for a constant gap height, the pumping velocity decreases relative to the maximum cylinder velocity as $K C$ increases. Thirdly, it can be readily shown that Eq. 7 has a local maximum during each half cycle, implying a maximum near bed velocity in space and time $\left(u_{\max }\right)$ during each up and down stroke of the cylinder.

Eq. 7 is used to predict the threshold of motion by assuming that (i) the peak seabed shear stress due to the pumping of fluid beneath the cylinder is related to $u_{\max }$ and (ii) that, although Eq. 7 implies that the near-bed velocity is not perfectly sinusoidal, the framework introduced by Soulsby (1997) may be used as a first estimate of the maximum shear stress. Adopting this approach, the maximum shear stress is then given by

$$
\tau_{\text {max }}=\frac{1}{2} \rho f_{w} u_{\text {max }}^{2}
$$

where $f_{w}$ is the (wave) friction factor and $u_{\max }$ is the maximum near-bed velocity amplitude. Following Soulsby (1997), the friction factor is calculated according to

$$
f_{w}=\max \left(f_{w, r o u g h}, f_{w, s m o o t h}\right)
$$

where

$$
f_{w, \text { rough }}=0.237 r^{-0.52}
$$

for rough turbulent flows, and:

$$
\begin{gathered}
f_{w, \text { smooth }}=2 R_{w}^{-0.5} \text { for } R_{w} \leq 5 \times 10^{5} \\
f_{w, \text { smooth }}=0.0521 R_{w}^{-0.187} \text { for } R_{w}>5 \times 10^{5}
\end{gathered}
$$

for laminar and smooth turbulent flows, respectively. In these equations $R_{w}$ represents the wave Reynolds number and is given by:

$$
R_{w}=\frac{u_{\max } A_{p}}{\nu}
$$

where $A_{p}$ is the half-cycle maximum particle excursion calculated by $A_{p}=u_{\max } T / 2 \pi$, and $r$ is a relative roughness given by:

$$
r=\frac{A_{p}}{k_{s}}
$$

where $k_{s}$ is the Nikuradse equivalent roughness of the bed, which is assumed to be equal to $2.5 d_{50}$.

Finally, to infer mobility, the shear stress computed from Eq. 8 can be compared with the critical shear stress for sediment transport. This is given in Table 1 for the present experimental sediment.

Figure 9 compares the measurements with estimates based on Eq. 7 to 13, shown by the blue shaded area bounding the predictions for the measured range in $h_{\min } / D$. The comparison indicates that the theoretical prediction works well in the region where the cylinder motions are consistent with the basic 
model assumptions (i.e. small $K C$ and $h_{m i n} / D$ ). When these assumptions are violated, sediment mobility is observed at different values of $\psi$ for a given $K C$ than predicted by the model. For instance, when $K C>4$ sediment mobility is observed at values of $\psi$ smaller than predicted theoretically. This implies an apparent amplification of the bed shear stress due to complex vortex-bed interactions. In other words, vortices increase the effectiveness of cylinder motion at initiating sediment transport. Similarly, at small $K C$ with larger $h_{\min } / D$, the agreement is poorer. This difference occurs because as $h_{\min } / D$ increases, relatively larger horizontal velocities occur near the cylinder than near the wall, which a depth-averaged velocity does not account for. Over-prediction of the near-wall velocity by continuity arguments, increasing with $h_{\min } / D$, is consistent with the findings of Tom (2018) comparing to numerical simulations.

\subsection{Extent of trench formation}

Table 2 lists the tests conducted where trench profiles were measured over at least 10,000 cylinder oscillations. For tests in Regime $1(K C<4)$ and Regime $3(K C>9)$ there was either no trench at the centreline of the cylinder, or the trench was non-uniform along the cylinder. However, for Regime 2 $(4<K C<9)$ a uniform trench was generally observed along the cylinder. For this regime, the time development of trench depth can be reasonably approximated by an exponential function of the form (see Figure 11 and 12):

$$
S(t)=S_{0}\left[1-\exp \left(-\left(\frac{N}{N_{t}}\right)^{n_{e}}\right)\right]
$$

where $S_{0}$ is the equilibrium trench depth, $N$ is the number of cycles, and both $N_{t}$ and $n_{e}$ are empirical coefficients controlling the rate of trench depth development and the non-linearity of the trench development during the initial stages. The results were fitted using a non-linear least-squares method and model coefficients are reported in Table 2. Although Eq. 14 describes trench depth progression well within the measured range of cycles, there is uncertainty in extrapolating to an equilibrium trench depth for some of the tests, particularly those with relatively low numbers of cycles and high $K C$. For these tests, the equilibrium trench depths may be less reliable but the fitted coefficients are still useful for comparing the trends over the initial $\mathcal{O}\left(10^{4}\right)$ cycles.

The results in Table 2 show that $n_{e}$ has little variation across the experiments, which implies a self-similar trench development in time. In contrast, there is larger variation in $S_{0}$ and $N_{t}$. To explore $S_{0}$, Figures 13 and 14 show the measured trench depths at $N=500,1,000,10,000$ and equilibrium conditions as a function of $K C$ and $\psi$. In agreement with the qualitative observations in Section 4, for each diameter of cylinder there is an evident increase in the trench depth with $K C$ and, to a lesser degree, $\psi$ for each number of cycles. However, there is variation for a constant $K C$ or $\psi$, and the trends (particularly for $\psi$ ) appear to vary with $\beta$ number. Closer inspection of the data on Figures 13 and 14 also indicates that over the range of experimental conditions tested the $K C$ number influences the trench depth at larger cycle numbers and at equilibrium conditions (compare Figure 13c and Figure 13d with Figure 13a), whereas $\psi$ influences the 


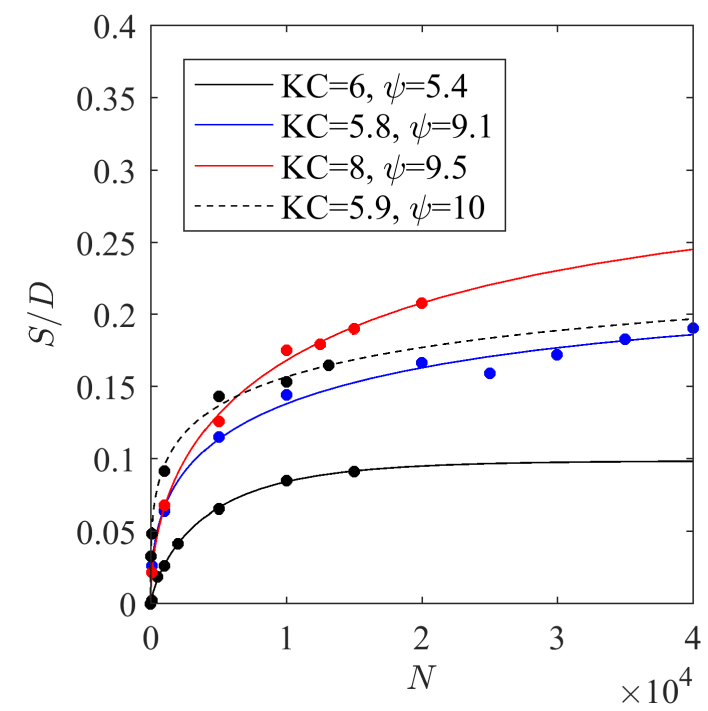

Fig. 11. Trench depth development. $D=21 \mathrm{~mm}(\beta=441$ to 630$)$

behaviour at relatively small cycle numbers (compare Figure 14a with Figure 14d). This result suggests that $\psi$ is most closely linked to the transport rate per cycle at the start of the trenching process, which would be expected to control the initial trench depth. In contrast, the $K C$ number appears to be a better predictor of the equilibrium trench depth. This implies that the equilibrium depth depends primarily on the scale of near-bed fluid motions.

To capture the combined influence of $K C$ and $\psi$ on trench depth, Figure 15 presents the trench depth as a function of both $K C$ and $\psi / \psi_{c r}$ for each cylinder diameter. The exponents $a$ and $b$ are selected to best collapse the data and $\psi_{c r}$ chosen based on the threshold of motion experiments for each $K C$ number. Provided both $K C$ and $\psi / \psi_{c r}$ are used in combination the trench depth data collapses well regardless of cycle number. Thus, for a given $\beta$ number order of magnitude and gap ratio, both $\psi$ and $K C$ affect the trenching process. Furthermore, as the cycle number increases $K C$ becomes more prominent in controlling the trench depth compared with $\psi$. Figure 15d similarly shows the fitted equilibrium trench depths as a function of $K C$ and $\psi / \psi_{c r}$ but with $a$ and $b$ chosen to correspond to the best fit results for $N=10,000$ (Figure 15c). These trends generally agree well, although there is some scatter due to uncertainty in fitting the equilibrium depth from a limited number of cycles. There does appear to be a $\beta$ dependency of the equilibrium results using this empirical fitting. However, the differences in equilibrium depth between across the range of $\beta$ considered are relatively small; and notably, there appears to be no significant difference between diameters for depth as a function of $K C$ number alone (Figure 13).

The equilibrium results from Figure 13d have been reproduced on Figure 16 with the best fit relationship 

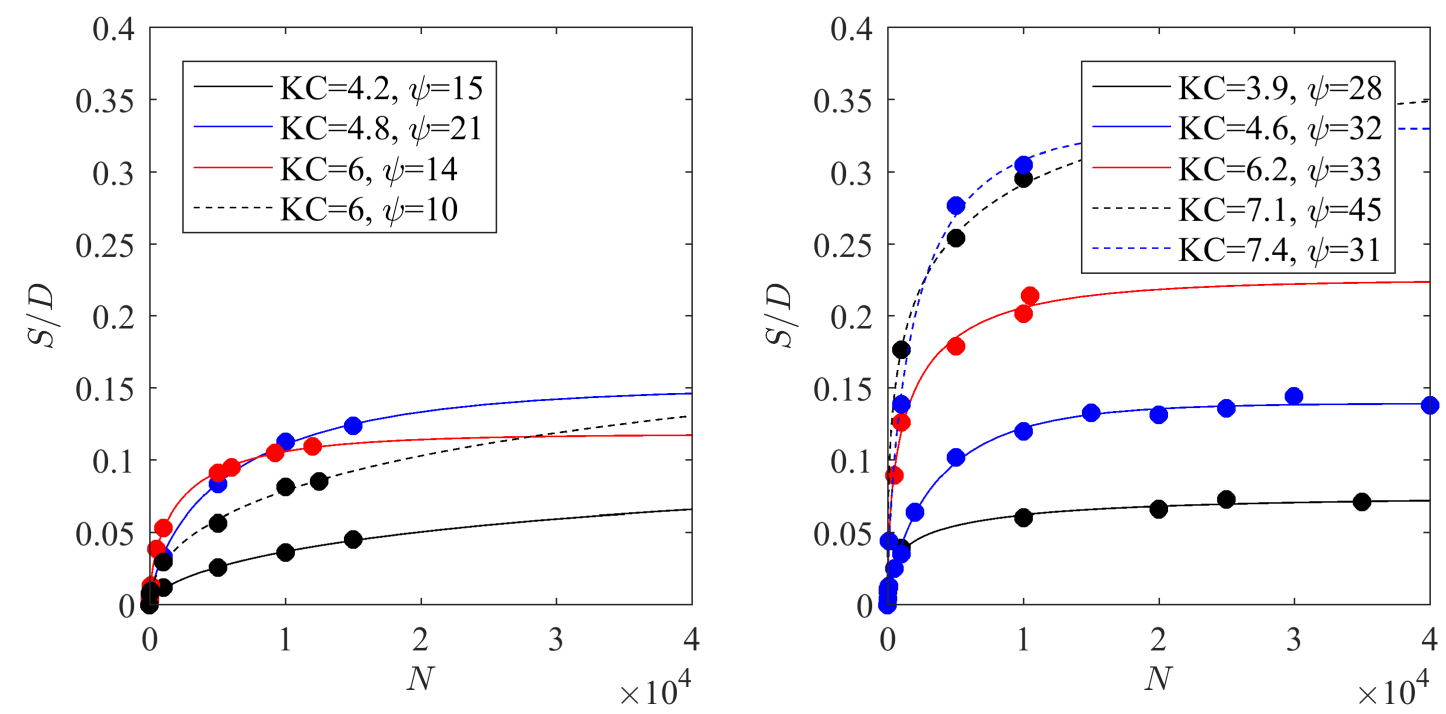

Fig. 12. Trench depth development. $D=51 \mathrm{~mm}(\beta=1,478$ to 3,716$)$

from Figure 13d corresponding to:

$$
\frac{S_{0}}{D}=0.1 K C-0.4 \text { for } 4<K C<9, \text { and } \psi / \psi_{c r}>3.0
$$

This fit implies no trench directly beneath the cylinder for $K C \leq 4$. The relationship is also limited to $K C<9$, due to the paucity of results at larger $K C$ and uncertainty related to changes in vortex shedding regimes as $K C$ increases beyond this range. The requirement for $\psi / \psi_{c r}>3.0$ reflects the range of parameters considered in the current expierments; for smaller $\psi / \psi_{c r}$ (particularly for $\psi \approx \psi_{c r}$ ), Eq. 15 would overestimate the trench depth. The results and fitted relationship are also compared with the empirical curve derived by Sumer and Fredsøe (1990) for scour below fixed pipelines in waves on Figure 16. The empirical result is based on experiments in live bed conditions (i.e. the motions are sufficiently strong to induce transport of sediment located far from the pipeline) and is in terms of a $K C$ number defined based on the orbital excursion of a water particle near the bed rather than the amplitude of the cylinder motion. At low $K C$ number, the equilibrium scour depth under a fixed cylinder is generally larger than that observed for the present experiments, regardless of mobility number. By contrast, the observed trench depths exceed the fixed pipe results at higher $K C$ numbers $(K C>6)$. This trend illustrates the difference in eroding mechanisms between fixed and oscillating cylinders. For a fixed cylinder, fluid motion is primarily parallel to the bed and is amplified beneath the cylinder, leading to mobilisation directly below the cylinder via flow amplification. This is in contrast to the oscillating cylinder, where the frequency and intensity of vortex-bed interactions drive erosion and appear to control trench depth. The results therefore indicate that the frequency and intensity of vortex-bed interactions scale differently with $K C$ number compared to 


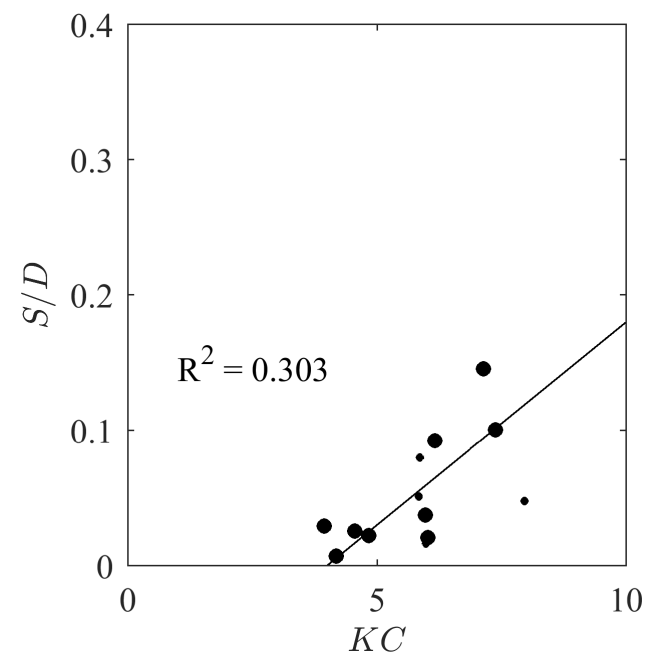

(a) $N=500$

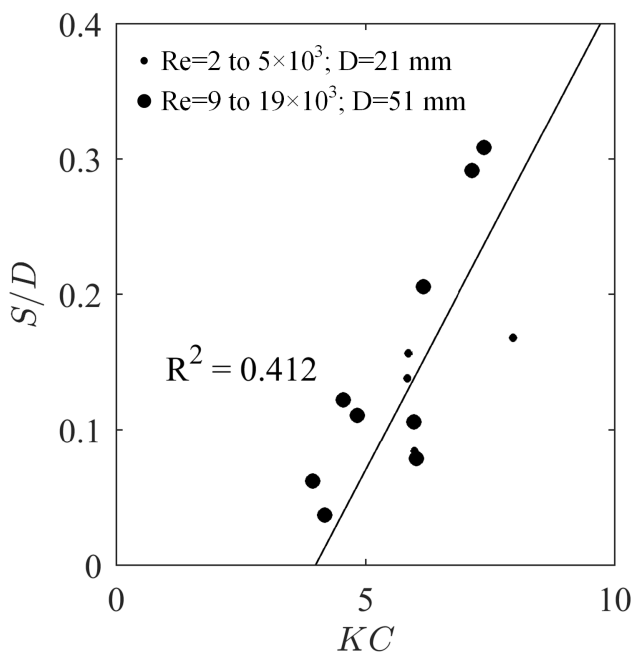

(c) $N=10,000$

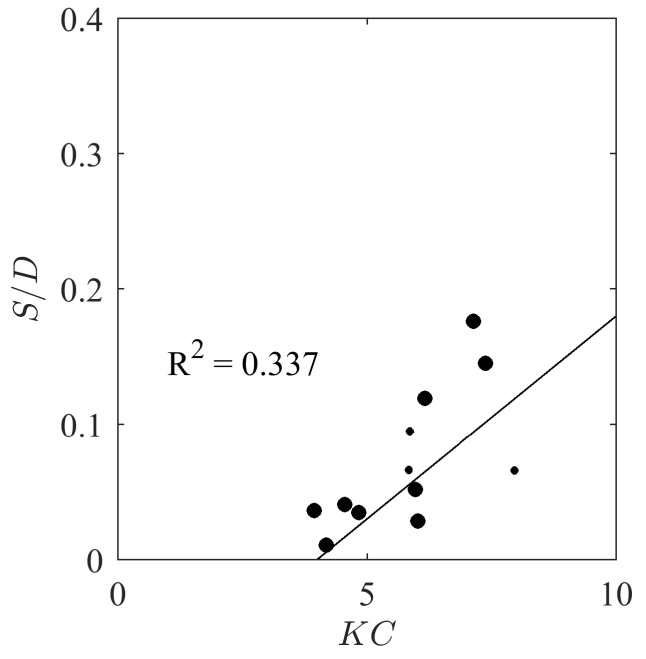

(b) $N=1000$

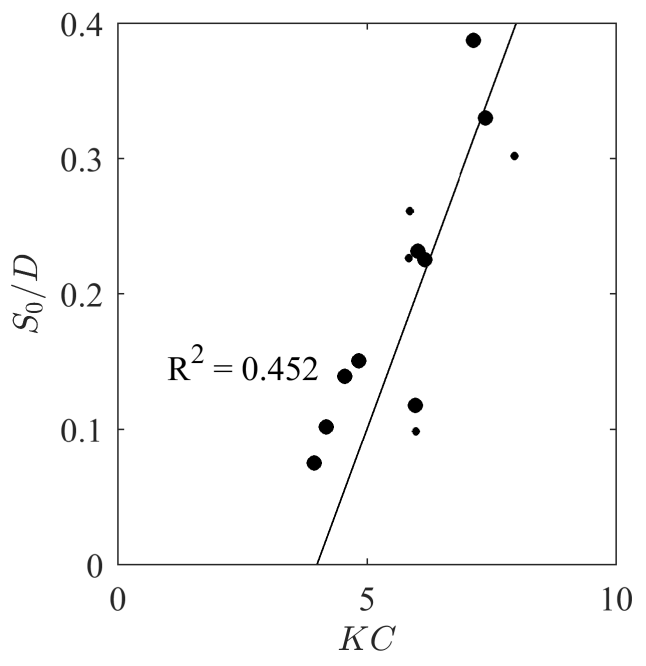

(d) $N=\infty$

Fig. 13. Variation in maximum trench depth for different numbers of cycles with $K C$. Fitted passing through $S / D=0$ at $K C=4$.

oscillatory flow under a fixed pipeline and imply that at least for $6 \lesssim K C<8$ the net transporting effect of vortices due to cylinder oscillation is larger than that due to amplification beneath a fixed cylinder.

\subsection{Initial rate of trench formation}

The initial rate of trench formation across all of the experiments can be compared to the derivative of Eq. 14, which is

$$
\frac{d S(t)}{d N}=S_{0}\left[n_{e} \frac{N^{n_{e}-1}}{N_{t}^{N_{e}}} \exp \left(-\left(\frac{N}{N_{t}}\right)^{n_{e}}\right)\right]
$$




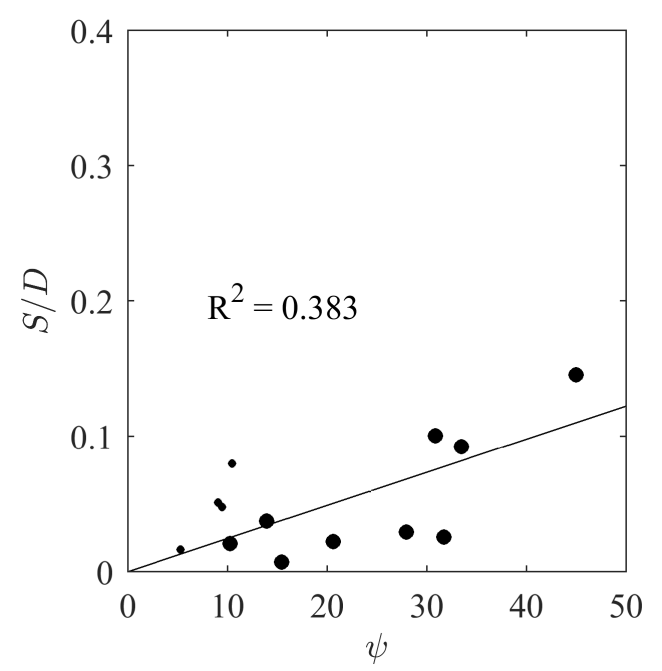

(a) $N=500$

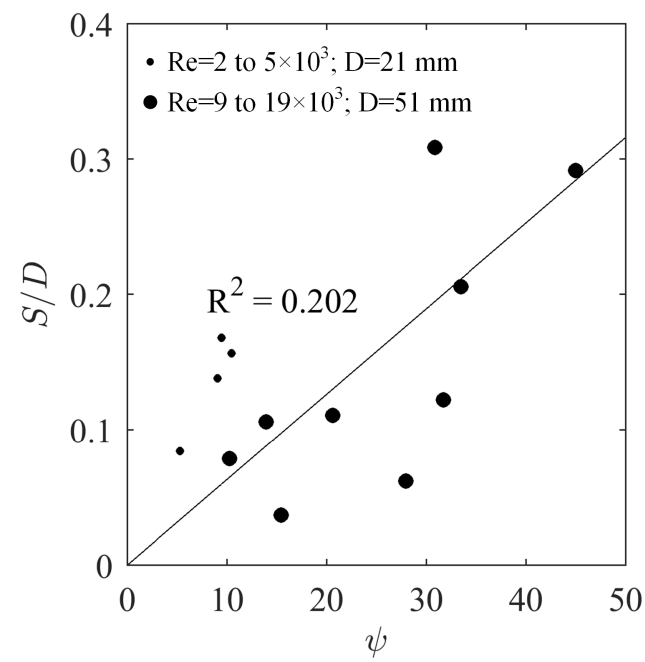

(c) $N=10,000$

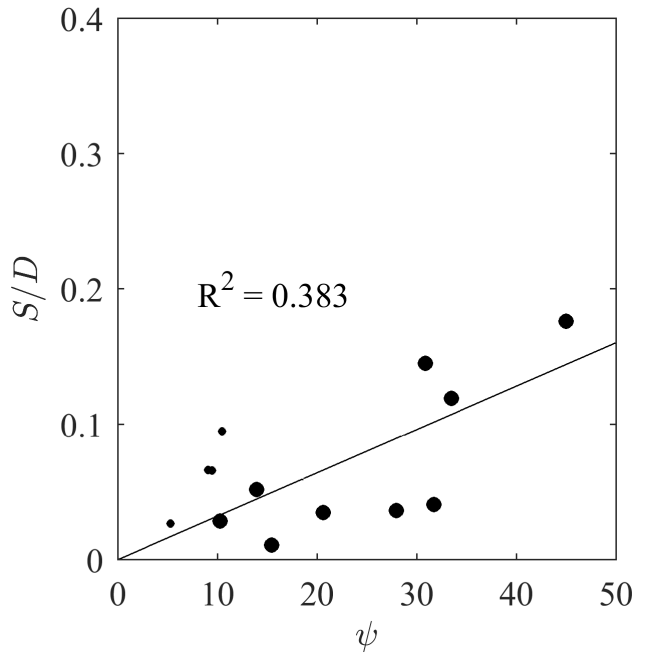

(b) $N=1000$

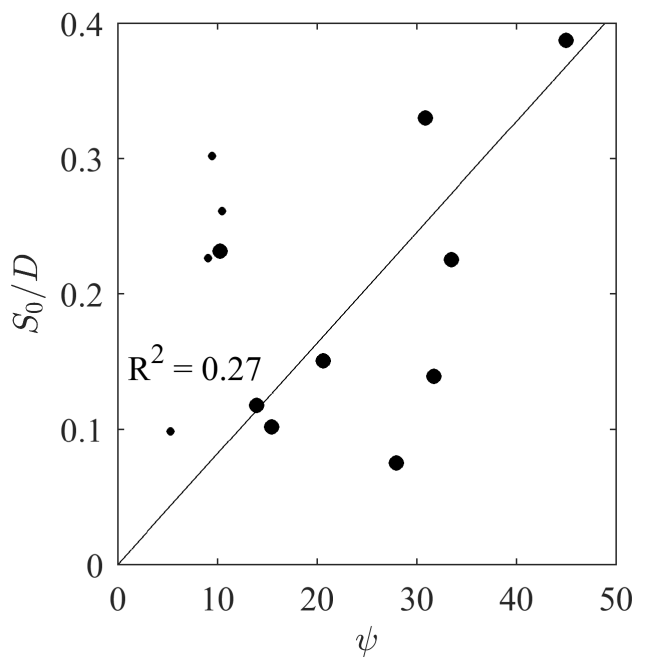

(d) $N=\infty$

Fig. 14. Variation in maximum trench depth for different numbers of cycles with $\psi$.

414

At $N=1$, since $N / N_{t} \ll 1$, this simplifies to

$$
\frac{d S(t)}{d N}=S_{0} \frac{n_{e}}{N_{t}^{n_{e}}}
$$

which describes the initial rate of trench development with cycles. For the current problem, such an eval-

more sensitive to the fitting method and varied more across the experiments. Since the influence of vortex impingements on bed shear stresses cannot be predicted accurately at present, methods to correct the initial rate, or $N_{t}$ and $n_{e}$, using an effective mobilised time approach (e.g. Larsen et al., 2017) have not been 


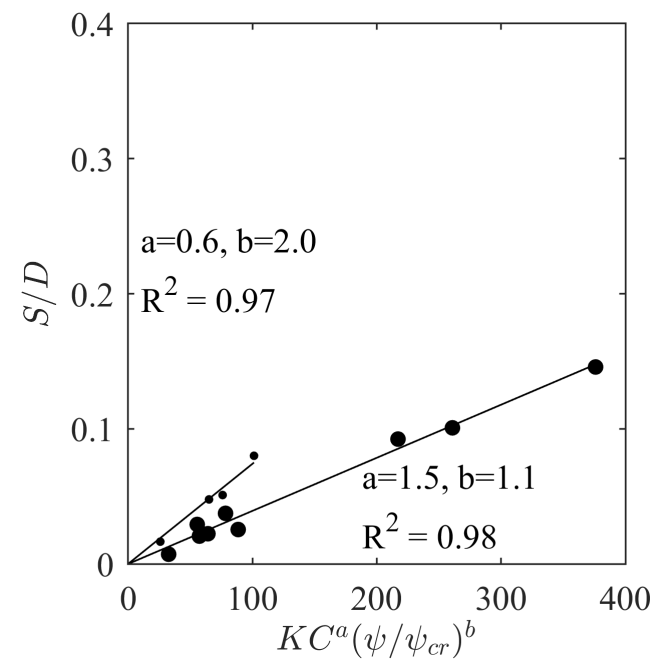

(a) $N=500$

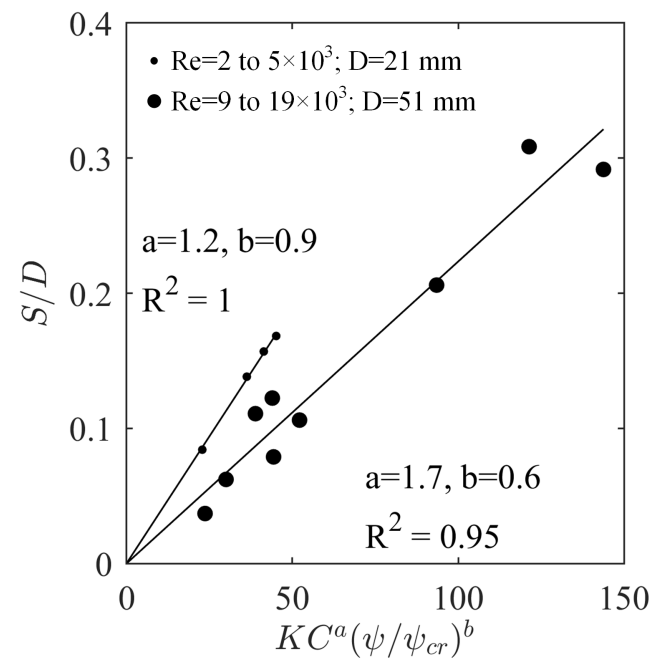

(c) $N=10,000$

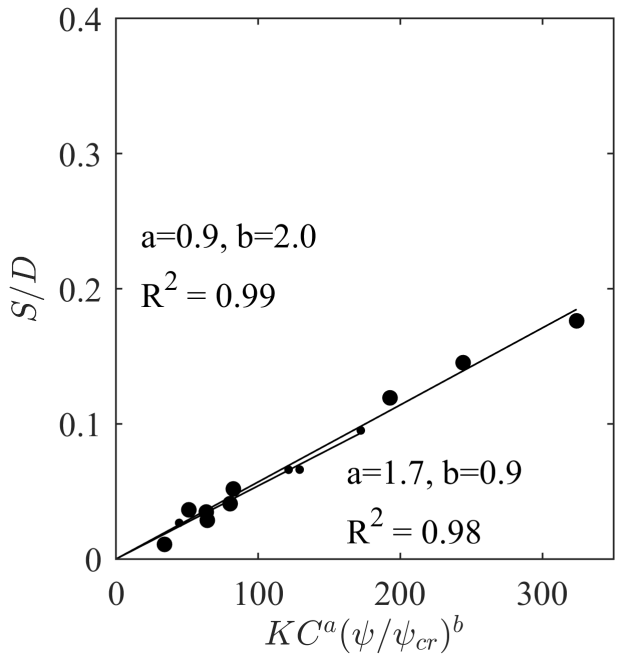

(b) $N=1000$

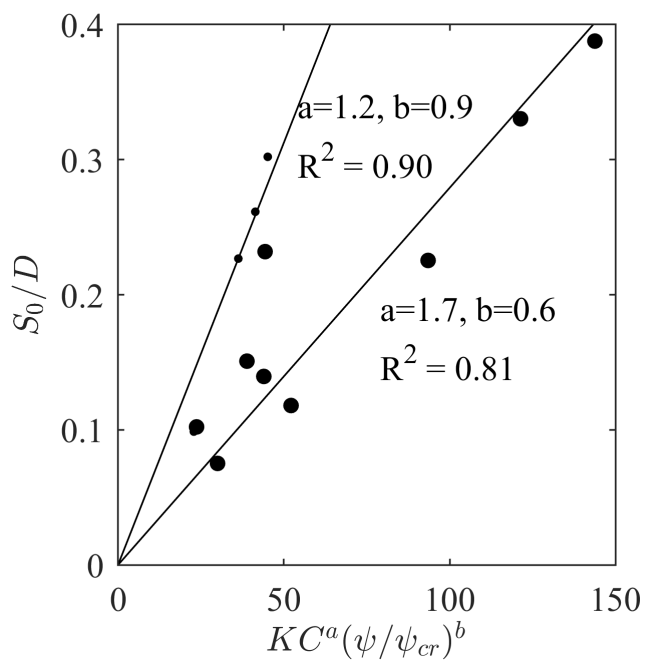

(d) $N=\infty$

Fig. 15. Trench depth at various cycle number as a function of $K C$ and $\psi / \psi_{c r}$.

attempted but would be an area of interest in further work.

Figure 17 shows the variation in initial rate of trenching as a function of $K C$ number and $\psi / \psi_{c r}$. There is scatter with respect to both parameters but a general trend of increasing rate with both $K C$ number and $\psi / \psi_{c r}$. These trends indicate that the near bed velocities and shear stresses due to vortex impingement events, which drive transport rates, increase with both $K C$ and $\psi / \psi_{c r}$. In general initial trench development rate increases at a faster rate with $\psi / \psi_{\text {cr }}$ for the smaller diameter cylinder, for which $\beta<700$, suggesting a dependency of the trench development rate on $\beta$. 


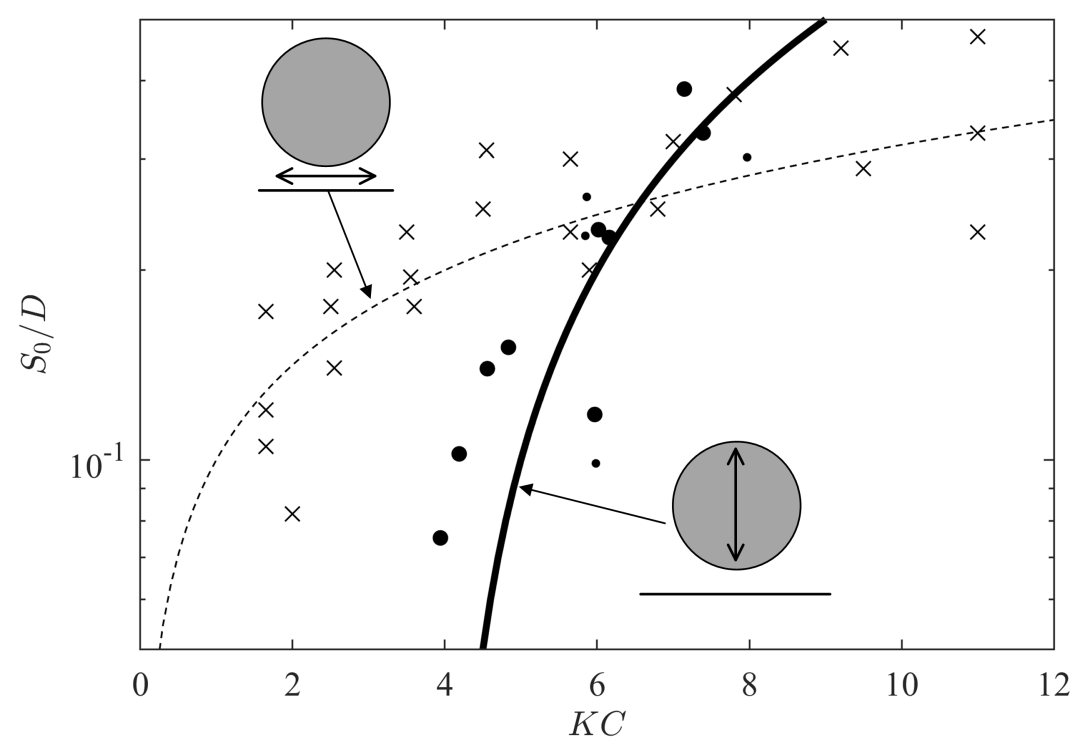

Fig. 16. Fitted equilibrium trench depths (solid circles - current study). Linear relationship corresponding to Eq. 15 shown as solid black line. Crosses - data reproduced from Sumer and Fredsøe (1990) and Lucassen (1984). Empirical relationship by Sumer and Fredsøe (1990) for pipeline scour beneath fixed pipelines in waves shown as dashed black line. $\psi / \psi_{\text {cr }}>3.0 .400<\beta<3800$.

\section{Discussion}

In this paper, sediment transport and trench development beneath an oscillating cylinder has been explored experimentally and shown to be driven by two main mechanisms, which depend primarily on the $K C$ number: (i) pumping of fluid in the gap leading to bedload transport and (ii) impingement of jet-like flows between vortices that occur as the cylinder moves away from the bed causing localised transport events. Observations indicate that a one-dimensional control volume model with an oscillatory boundary layer assumption predicts $\psi$ required for sediment motion at low $K C$ and for small $h_{\min } / D$ when pumping dominates. At larger $K C$ numbers, vortex-bed interactions cause sediment motion to occur at relatively smaller $\psi$ values than predicted by the one-dimensional model.

The trench depth and initial growth rate trends appear proportional to both $K C$ and $\psi$, with a stronger dependency of equilibrium trench depth on $K C$. For $K C<9$, Figure 16 indicates that the relationship between $K C$ and $S_{0} / D$ given by Eq. 15 provides an estimate of the trench depth, encompassing the variation across $400<\beta<3800$, which covers the lower range of typical field conditions described in Section 3. Consequently, Eq. 15 may be used to predict the equilibrium trench depth in the field, provided $\psi / \psi_{c r} \gg 1$ (i.e. sediment is mobilised). For motions where $\psi / \psi_{c r} \approx 1$, Eq. 15 is likely to represent an upper bound estimate. 


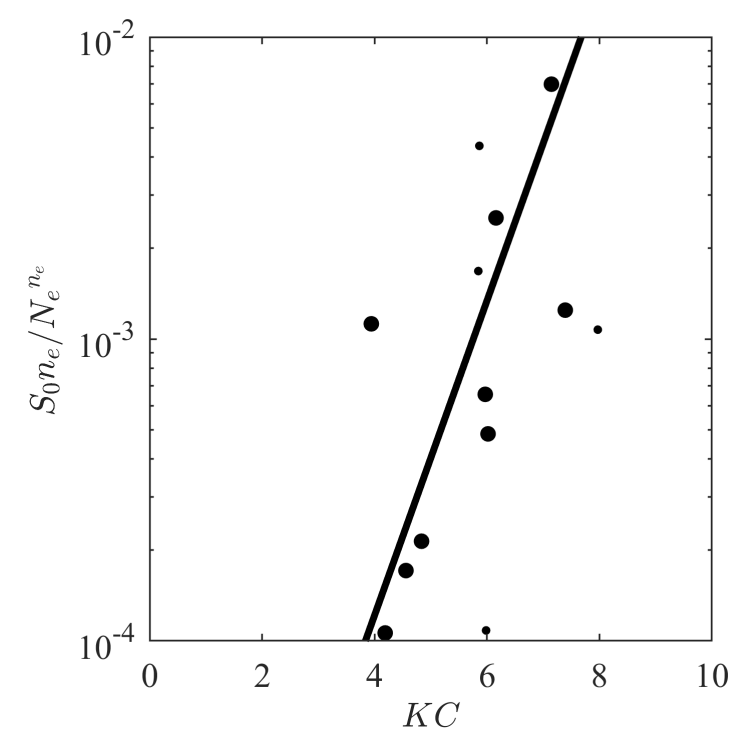

(a) Function of $K C$ number

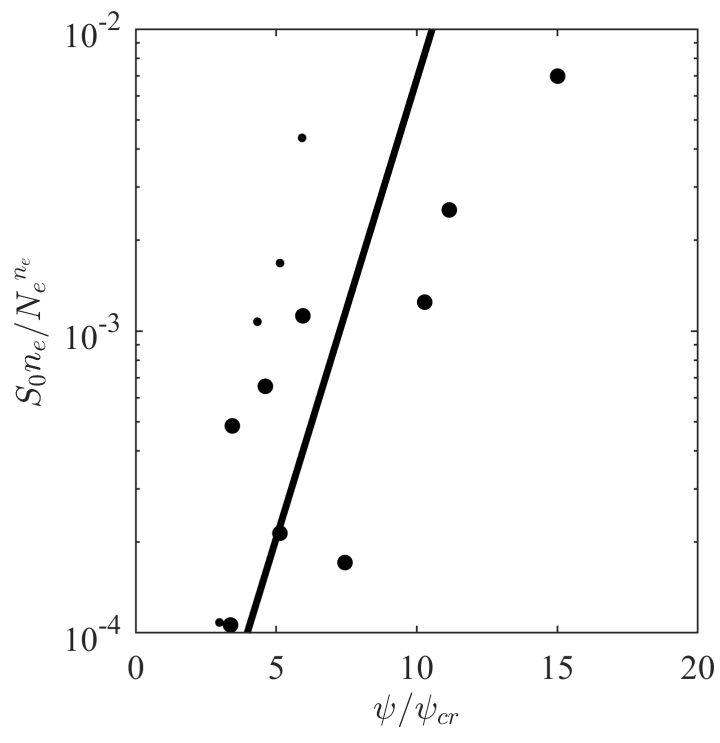

(b) Function of $\psi / \psi_{c r}$

Fig. 17. Inferred initial rates of trench development. Marker size indicates diameter of cylinder used in the experiments.

An important aspect of the experiments presented in this paper is that only the effect of cylinder motion on transport and trench development has been considered. This was done to deliberately isolate the effect of fluid-structure interaction (i.e. the induced flow field) from the effects of structure-seabed interaction. The results show that trenches up to $0.4 D$ can form without structure-seabed interaction, provided that $\psi$ is sufficiently large for sediment mobility to occur; but the resulting trench geometry depends on $K C$ number. For $K C<4$, net transport over multiple cycles forms a prominent central ridge beneath the cylinder due to symmetry. In practice, such ridge formation may be hindered due to structure-seabed interactions if vertical motion amplitudes vary leading to the cylinder plastically deforming the ridge material. At larger $K C$ numbers, sediment mobility is enhanced by vortex-seabed interaction; but for $K C<9$ transport due to vortices occurs on both sides of the cylinder causing an eventually symmetric trench extending below the initial bed level beneath the cylinder. The vortices enhance the peak seabed flow velocities, and therefore the growth rate and final depth of trenches, and continuity arguments alone are not able to explain the increases. Empirical results offer an initial estimate of the likelihood of trench formation and both the depth and rate of trench formation at higher $K C$.

To fully model the behaviour of an oscillating near-seabed structure, there is a need to incorporate structure-seabed interaction either experimentally or numerically. However, with respect to numerical modelling, the complexities of the transport mechanisms observed in this work suggest that if computational 


\section{Conclusions}

fluid dynamics simulations are used to explore this then they must be able to correctly simulate the vortex dynamics induced by cylinder motion (for $K C>4$ ). The fluctuating vortex dynamics on a cycle by cycle basis are important to resolve to capture the eventual symmetry of the trench profile (for Regime 2) and detailed time development of trenching. For higher $K C$ (i.e. $>9$ ), the trench observations showed significant three-dimensional effects, which suggests the need to capture three-dimensional flows in any numerical modelling and to ensure that an experimental facility is adequate to avoid (or quantify) end effects.

In the context of previous literature, the results suggest that although the current phenomena are ostensibly similar to wave-induced scour of fixed pipelines, there are key differences in the mechanisms of sediment transport for each scenario. For a fixed pipeline, the funnelling of externally applied flows through a narrow gap beneath the cylinder amplifies the near-bed velocity and applied bed shear above those felt elsewhere along the bed away from the cylinder. This has the capacity to transport sediment relatively far from the pipeline. In contrast, for a normally oscillating cylinder, the near-bed pumping velocity beneath the cylinder for $h_{\min } / D=0.125$ is on the order of the maximum cylinder velocity for $K C<4$ (see Eq. 7 and Tom, 2018). For this reason, the trench depths for an oscillating cylinder are less than for a fixed cylinder with an equivalent $K C \lesssim 6$. However, when vortices enhance the near-bed fluid motions relative to pumping flow at larger $K C$, trench development intensifies as a stronger function of $K C$.

The relatively symmetric equilibrium trench geometries identified for Regime 2 are consistent with the findings of Chiew et al. (2016), who showed similarly shaped and sized trenches beneath an elastically mounted cylinder that was allowed to contact the bed and had an approximate $K C$ number of 8 to 9 . Chiew et al. (2016) note that vortex interactions drive the transport, which is in agreement with the findings in the present work. They also suggest that the flow field, when ensemble-averaged, is symmetric about the cylinder and that this symmetry leads to the symmetric trench shape. With respect to the latter, the observations in the current body of work indicate that the evolution of the trench during individual cycles is driven by instantaneously asymmetric transport even though the trench shape eventuates to become symmetric. Hence, although when averaged over many cycles, the flow field appears symmetric (Tom, 2018), this is only because the flow asymmetry varies frequently between cycles and along the cylinder for $4<K C<9$. The current findings expand on the work by Chiew et al. (2016) by specifically focusing on the bed dynamics that result without contacting the bed and the physics that drive trenching over a larger parameter space than previously explored. The understandings gained from these observations provide a phenomenological basis to assess trench development for a number of applications. The $K C$ number range considered covers practical applications ranging from pipelines undergoing lay installation to operational motions of SCRs.

The main conclusions from this study are: 
1. The sediment transport behaviour beneath oscillating cylinders in otherwise still water is governed by the near-bed flow field, which varies as a function of $K C, \psi$, and $h_{m i n} / D$. For the range of cylinder motions and the sediment considered, trench development occurs local to the cylinder and progresses quickly during the initial stages of cycling before becoming increasingly slow as the trench develops towards an equilibrium.

2. The influence of vortex shedding is found to enhance the trench extent and growth rate, relative to continuity-driven pumping flow.

3. For $K C<12$ three regimes were identified in the transport behaviour and trench formation:

(a) $K C \lesssim 4$ where the flow and transport is generally symmetric and driven by pumping of fluid in the gap between the cylinder and bed. A prominent central ridge with two parallel trenches forms in this regime.

i. Control volume arguments and an oscillatory boundary layer assumption to infer the bed shear stress provide reasonable agreement with the measured $\psi$ required to mobilise sediment at a given $K C$ number. This agreement reduces as $h_{\min } / D$ increases.

(b) $4 \lesssim K C \lesssim 9$ where the flow and transport is periodically asymmetric over tens of cycles and localised transport events attributed to jet-like flow between detached vortices becomes increasingly important in driving transport. The trench profiles in this regime are transiently asymmetric but generally tend towards a symmetric equilibrium.

i. Simple control volume arguments are not able to predict sediment motion due to the influence of detached vortices, which increase in intensity with both $K C$ and $\psi$. Control volume arguments generally underpredict the potential for sediment motion for these $K C$ numbers.

ii. For $N<10,000$ the trench depths and initial growth rates increase as functions of both $K C$ and $\psi / \psi_{c r}$. At small cycle numbers, $\psi / \psi_{c r}$ primarily controls; and $K C$ number increases in importance with cycling.

iii. Equilibrium trench depth is found to be a function (primarily) of $K C$ number and found to be reasonably represented by Eq. 15 for $K C<9$ and $\psi / \psi_{c r}>3.0$.

iv. The magnitude of trench depths (relative to the cylinder diameter) is similar for both cylinder diameters; however, there appears to be a dependency of equilibrium trench depth and rate of trench formation on $\beta$ when both $K C$ and $\psi / \psi_{c r}$ are considered. This dependency requires further investigation to validate these results for use at different $\beta$ in practice.

(c) $K C \gtrsim 9$ where the flow and transport are predominantly asymmetric. The direction of the asymmetry switches sides over the course of relatively long time periods (tens to hundreds of 
cycles), and the trench profiles are significantly asymmetric. The direction of trench asymmetry can still vary over many cycles.

\section{Acknowledgements}

This work was funded by research and development grants from the University of Western Australia (UWA) and the ARC Industrial Transformation Research Hub for Offshore Floating Facilities, which is funded by the Australian Research Council, Woodside Energy, Shell, Bureau Veritas and Lloyds Register (Grant No. IH140100012). The authors would also like to thank Ian Milne and Liang Cheng for helpful comments during the preparation of this paper and Henning Mohr for assistance with the experiments presented in this paper.

\section{References}

Bhattacharjee, S., Majhi, S., Smith, D., Garrity, R., 2014. Serpentina fpso mooring integrity issues and system replacement: Unique fast track approach. In: Offshore Technology Conference. Offshore Technology Conference.

Bridge, C., 2005. Effects of seabed interaction on steel catenary risers. Ph.D. thesis, University of Surrey.

Bridge, C. D., Howells, H. A., 2007. Observations and modeling of steel catenary riser trenches. In: The Seventeenth International Offshore and Polar Engineering Conference. International Society of Offshore and Polar Engineers.

Chiew, Y., Hsieh, S., Low, Y., 2016. Characteristics of scour and flow field beneath a forced vibrating circular cylinder. In: Scour and Erosion: Proceedings of the 8th International Conference on Scour and Erosion (Oxford, UK, 12-15 September 2016). CRC Press, p. 171

Chiew, Y., Hsieh, S., Yeh, P., Low, Y., Li, F., 2014. Local scour and flow characteristics around a vibrating catenary riser. In: Proceedings Seventh International Conference on Scour and Erosion. pp. 685-691.

Elliott, B. J., Zakeri, A., Barrett, J., Hawlader, B., Li, G., Clukey, E. C., 2013. Centrifuge modeling of steel catenary risers at touchdown zone part ii: Assessment of centrifuge test results using kaolin clay. Ocean Engineering 60, $208-218$.

Gao, F.-P., Yang, B., Wu, Y.-X., Yan, S.-M., 2006. Steady current induced seabed scour around a vibrating pipeline. Applied Ocean Research 28 (5), 291-298.

Hejazi, R., 2018. Personal communication.

Hughes, S. A., 1993. Physical models and laboratory techniques in coastal engineering. Vol. 7. World Scientific.

Larsen, B. E., Fuhrman, D. R., Baykal, C., Sumer, B. M., 2017. Tsunami-induced scour around monopile foundations. Coastal Engineering 129, 36-49.

Leckie, S. H., Mohr, H., Draper, S., McLean, D. L., White, D. J., Cheng, L., 2016. Sedimentation-induced burial of subsea pipelines: Observations from field data and laboratory experiments. Coastal Engineering 114, 137-158.

Lucassen, R., 1984. Scour underneath submarine pipelines. TU Delft, Civil Engineering and Geosciences, Hydraulic Engineering.

Mohr, H., Draper, S., Cheng, L., White, D., 2016. Predicting the rate of scour beneath subsea pipelines in marine sediments under steady flow conditions. Coastal Engineering 110, 111-126.

Soulsby, R., 1997. Dynamics of marine sands: a manual for practical applications. Thomas Telford.

Sumer, B., Jensen, B., Fredsøe, J., 1991. Effect of a plane boundary on oscillatory flow around a circular cylinder. Journal of Fluid Mechanics 225, 271-300.

Sumer, B. M., Fredsøe, J., 1990. Scour below pipelines in waves. Journal of waterway, port, coastal, and ocean engineering $116(3), 307-323$. 
Sumer, B. M., Fredsøe, J., 2002. The mechanics of scour in the marine environment. World Scientific.

Sumer, B. M., Fredsøe, J., 2006. Hydrodynamics around cylindrical strucures. Vol. 26. World Scientific.

Sumer, B. M., Mao, Y., Fredsøe, J., 1988. Interaction between vibrating pipe and erodible bed. Journal of Waterway, Port, Coastal, and Ocean Engineering 114 (1), 81-92.

Tatsuno, M., Bearman, P. W., 1990. A visual study of the flow around an oscillating circular cylinder at low keulegan-carpenter numbers and low stokes numbers. Journal of Fluid Mechanics 211 (1), 157.

Tom, J. G., 2018. Fluid-pipeline-soil interaction at the seabed. Ph.D. thesis, University of Western Australia.

Westgate, Z., Randolph, M., White, D., Li, S., 2010a. The influence of sea state on as-laid pipeline embedment: a case study. Applied Ocean Research 32 (3), 321-331.

Westgate, Z., White, D., Randolph, M. F., Brunning, P., et al., 2010b. Pipeline laying and embedment in soft fine-grained soils: field observations and numerical simulations. In: Offshore Technology Conference. Offshore Technology Conference.

Williamson, C., 1985. Sinusoidal flow relative to circular cylinders. Journal of Fluid Mechanics 155, $141-174$. 